\title{
Complémentarité des techniques RMN, IQNS et des simulations numériques pour l'étude des mouvements moléculaires
}

\author{
F. Guillaume
}

\author{
Laboratoire de Physico-Chimie Moléculaire, UMR 5803 du CNRS, Université de Bordeaux I, \\ 351 cours de la Libération, 33405 Talence cedex, France
}

\begin{abstract}
Résumé:
Le but de cet article est de souligner l'intérêt de combiner les techniques de la spectroscopie de diffusion incohérente quasi-élastique des neutrons (IQNS), de la résonance magnétique du deutérium (RMN ${ }^{2} \mathrm{H}$ ) et des simulations numériques (MD) pour analyser les fluctuations Browniennes de molécules organiques en phase condensée. Les principes des techniques IQNS et MD étant discutés et illustrés dans d'autres articles publiés dans ce journal consacré à l'école thématique "diffusion quasi-élastique des neutrons", nous insistons plus particulièrement sur les principes de la spectroscopie $\mathrm{RMN}{ }^{2} \mathrm{H}$. Nous discutons de la complémentarité de ces techniques et illustrerons par quelques exemples comment il est possible de déterminer le potentiel de force moyenne "local" dans des cristaux organiques.
\end{abstract}

\section{INTRODUCTION}

Toute technique spectroscopique permet en principe de caractériser les fluctuations Browniennes de translation et/ou de rotation moléculaire en phase condensée et à l'équilibre thermodynamique. Pour des solides cristallins ou des cristaux liquides (ou d'autres milieux anisotropes), les techniques de diffusion quasi-élastique incohérente des neutrons (IQNS) et de résonance magnétique nucléaire du deutérium $\left({ }^{2} \mathrm{H}\right.$ RMN) sont parmi les plus utilisées. Nous allons montrer pourquoi il est souvent nécessaire de combiner les techniques IQNS, ${ }^{2} \mathrm{H}$ RMN et les simulations numériques de la dynamique moléculaire (MD) pour déterminer avec précision les mouvements "individuels" de rotation (et/ou de translation) de molécules organiques dans des phases solides.

Pourquoi plusieurs méthodes d'investigation pour comprendre un même phénomène? En général les mouvements de diffusion en phase condensée sont relativement lents et se manifestent par des modifications subtiles des profils spectraux. On est alors bien souvent amené à proposer des modèles rendant compte de ces modifications spectrales et ces modèles doivent bien entendu être simples et comporter peu de paramètres. Il n'est pas rare, pour ne pas dire fréquent, que des modèles assez différents les uns des autres puissent être appliqués avec un égal bonheur pour analyser les données expérimentales. Nous verrons par exemple que des modèles de rotations uniaxialles par sauts sur $N$ sites (avec $N \geq 3$ ) conduisent tous (quel que soit $\mathrm{N}$ ) exactement aux même profils $\mathrm{RMN}{ }^{2} \mathrm{H}$. Il n'est pas rare qu'un modèle dynamique rende compte de façon satisfaisante des spectres IQNS et que ce même modèle échoue complètement pour rendre compte des expériences $\mathrm{RMN}{ }^{2} \mathrm{H}$ (et vice-versa). Il est également souvent indispensable de procéder à une estimation préalable des échelles de temps caractéristiques de la dynamique moléculaire pour guider l'utilisateur dans le choix du spectromètre de diffusion neutronique approprié. La RMN du deutérium permet de réaliser assez facilement ces estimations préliminaires. Il nous semble important de rendre plus systématiques des méthodes d'analyses multi-techniques. Pour cela, il convient d'adopter un formalisme permettant d'élaborer des modèles dynamiques qui soient les mêmes quelle que soit la technique expérimentale considérée. Nous verrons que pour des cas complexes les simulations $\mathrm{MD}$ vont nous aider considérablement pour l'élaboration de ces modèles.

Pour simplifier cette comparaison entre les techniques IQNS et RMN ${ }^{2} \mathrm{H}$, nous allons nous contenter de discuter de mouvements de rotation uniaxiale dans des cristaux moléculaires. Nous présenterons dans 
une première partie le formalisme général nous permettant de proposer des modèles dynamiques indépendamment de toute technique. Dans une seconde partie, nous présenterons la théorie sommaire des techniques IQNS et $\mathrm{RMN}{ }^{2} \mathrm{H}$ en essayant de montrer comment les résultats expérimentaux peuvent être analysés à partir des modèles dynamiques présentés auparavant. Nous insisterons plus particulièrement, dans le cadre de cette école thématique, sur la $\mathrm{RMN}^{2} \mathrm{H}$. Nous illustrerons la complémentarité de ces techniques à l'aide de deux exemples, le cristal de benzène chrome tricarbonyle et le cristal de $n$ nonadécane.

\section{MODELES}

Toute analyse de profil spectral repose sur l'élaboration d'un modèle dynamique permettant de calculer des spectres que l'on peut comparer à l'expérience. Des modèles analytiques simples pour décrire les translations et les rotations moléculaires existent depuis longtemps, à travers l'étude du mouvement Brownien de translation ou de rotation (équations de Langevin et de Fokker-Planck). Nous n'allons rappeler ici que les principes permettant de proposer des modèles pour le mouvement Brownien de rotation uniaxiale.

Pour les spectroscopies IQNS ou RMN $2 \mathrm{H}$, le problème consiste à déterminer la probabilité conditionnelle $\mathbf{p}\left(\phi ; \phi_{0}\right)$ de trouver une liaison C-H ou C-D dans une orientation $\phi$ à l'instant $t$ sachant que son orientation était $\phi_{0}$ à l'instant initial $\mathrm{t}_{0}$. Cette probabilité conditionnelle est obtenue par résolution de l'équation de Fokker-Planck, par exemple pour une rotation uniaxiale:

$$
\frac{\partial}{\partial \mathrm{t}} \mathrm{p}\left(\phi, \mathrm{t} ; \phi_{0}, 0\right)=\Re(\phi) \mathrm{p}\left(\phi, \mathrm{t} ; \phi_{0}, \mathrm{t}_{0}\right)
$$

où $\Re(\phi)$ est l'opérateur de Fokker-Planck:

$$
\Re(\phi)=D_{\mathrm{r}}\left(\frac{\partial^{2}}{\partial \phi^{2}}+\beta \frac{\partial \mathrm{V}(\phi)}{\partial \phi} \frac{\partial}{\partial \phi}+\beta \frac{\partial^{2} \mathrm{~V}(\phi)}{\partial \phi^{2}}\right)
$$

$D_{r}$ est le coefficient de diffusion rotationnelle, $V(\phi)$ le potentiel de force moyenne. La fonction de distribution des orientations $\mathrm{P}(\phi)$ à l'équilibre thermodynamique est donnée par la relation:

$$
\mathrm{P}(\phi)=\frac{1}{\mathrm{~F}} \exp (-\beta \mathrm{V}(\phi))
$$

où $\mathrm{F}$ est un facteur de normalisation. A l'exception de quelques cas simples, il n'y a en général pas de solution analytique à l'équation de Fokker-Planck et des approches numériques sont nécessaires. Nous allons donc considérer une fonction de distribution $P(\phi)$ discrète définie sur $N$ points. Les dérivées apparaissant dans l'équation de Fokker Planck sont calculées par différences finies de sorte que nous sommes conduits à résoudre un système de $\mathrm{N}$ équations différentielles de la forme:

$$
\left\|\frac{\mathrm{d}}{\mathrm{dt}} \mathrm{p}_{\mathrm{i}}\right\|=\left\|\mathrm{L}_{\mathrm{ij}}\right\|\left\|\mathrm{p}_{\mathrm{i}}\right\|
$$

où $p_{\mathrm{i}}$ représente la probabilité à l'instant $\mathrm{t}$ que la molécule ait l'orientation i et $\left\|\mathrm{L}_{\mathrm{ij}}\right\|$ est la matrice carrée $\mathrm{NxN}$ dont les éléments $\mathrm{L}_{\mathrm{ij}}$ sont donnés par [1]: 


$$
L_{i j}=\left\{\begin{array}{cc}
-\left(L_{i-1, j}+L_{i+1, j}\right) & \text { pour } j=i \\
D_{r} \delta^{-2} \sqrt{\frac{P\left(\phi_{i}\right)}{P\left(\phi_{i \pm 1}\right)}} & \text { pour } j=i \pm 1 \\
0 \quad \text { pour les } & \text { autres valeurs }
\end{array}\right.
$$

et les probabilités conditionnelles:

$$
p\left(\phi_{j}, t ; \phi_{i}, 0\right)=\sum_{n=1}^{N}\left[x_{j n}\right]^{-\tau_{n}^{-1}|t|}\left[x_{n i}\right]^{-1}
$$

où $\delta$ est le pas choisi dans la discrétisation de $\mathrm{P}(\phi),\left\lfloor\mathrm{X}_{\mathrm{jn}}\right\rfloor$ représente l'élément de la matrice des vecteurs propres (rangés en colonnes) et $\tau_{\mathrm{n}}^{-1}$ la valeur propre associée de la matrice $\left\|\mathrm{L}_{\mathrm{ij}}\right\|$. Ce formalisme est similaire à celui présenté G. Coddens [2].

Une fois les probabilités conditionnelles connues, des fonctions de corrélation puis des profils spectraux (par transformation de Fourier) sont calculés. L'analyse des spectres se fera alors selon le principe de l'ajustement des profils théoriques aux données expérimentales en faisant varier les paramètres du modèle que sont $D_{r}$ et ceux qui définissent $P(\phi)$. Une difficulté évidente est la détermination de la fonction de distribution des orientations $\mathrm{P}(\phi)$. Pour un cristal, des considérations de symétrie peuvent suffire. Remarquons cependant que cette fonction est obtenue directement par des simulations numériques de la dynamique moléculaire.

\section{METHODES}

\subsection{Spectroscopie IQNS}

La théorie de la diffusion incohérente quasi-élastique des neutrons (IQNS) a été présentée par M. Bée [3] et P. Damay [4]. Nous ne ferons ici que quelques rappels succincts. Pour un échantillon composé de molécules hydrogénées, la diffusion quasiélastique incohérente des neutrons dépend du produit scalaire du vecteur transfert de moment $\vec{Q}$ par le vecteur déplacement $(\vec{r}(t)-\vec{r}(0))$ d'un atome d'hydrogène, selon la loi de diffusion intermédiaire (dans la limite classique):

$$
\mathrm{I}(\overrightarrow{\mathrm{Q}}, \mathrm{t})=\left\langle\mathrm{e}^{\mathrm{i} \overrightarrow{\mathrm{Q}} \overrightarrow{\mathrm{r}}(\mathrm{t})} \mathrm{e}^{-\mathrm{i} \overrightarrow{\mathrm{Q}} \overrightarrow{\mathrm{r}}(0)}\right\rangle
$$

Dans la fenêtre spectrale correspondant à la diffusion quasiélastique (transferts d'énergie $<3 \mathrm{meV}$ ), la loi de diffusion traduira les dynamiques de rotation et de translation des atomes d'hydrogène. Dans la limite "statique", c'est à dire lorsque les mouvements sont "gelés", le spectre est purement élastique et ne fournit aucune information sur les positions atomiques (en diffusion incohérente). L'analyse des profils IQNS repose sur des procédures d'ajustement d'une fonction théorique sur les spectres expérimentaux:

$$
S(\vec{Q}, \omega)=\exp (-2 W(\vec{Q}))\left[S^{t}(\vec{Q}, \omega) \otimes R(Q, \omega)+\left(a+b Q^{2}\right)\right]
$$


$\overrightarrow{\mathrm{Q}}$ est le transfert de moment à l'angle de diffusion $2 \theta$ et à l'énergie $\hbar \omega$ de module:

$$
\mathrm{Q}=\frac{2 \pi}{\lambda_{0}} \sqrt{2+\frac{\hbar \omega}{\mathrm{E}_{0}}-2\left(1+\frac{\hbar \omega}{\mathrm{E}_{0}}\right)^{1 / 2} \cos (2 \theta)}
$$

(où $\lambda_{0}$ est la longueur d'onde du faisceau incident et $E_{0}$ l'énergie correspondante) et l'orientation de $\vec{Q}$ par rapport à la direction de propagation du faisceau incident est donnée par l'angle $\Phi$ :

$$
\cos \Phi=\frac{\lambda_{0} \mathrm{Q}}{4 \pi}-\frac{\pi}{\lambda_{0} \mathrm{Q}} \frac{\hbar \omega}{\mathrm{E}_{0}}
$$

Dans l'équation (8), $\exp (-2 \mathrm{~W}(\overrightarrow{\mathrm{Q}}))$ est le facteur de Debye-Waller, $\mathrm{Q}_{0}$ est le transfert de moment élastique, $\mathrm{R}(\mathrm{Q}, \omega)$ est la fonction de résolution instrumentale et $S^{t}(\vec{Q}, \omega)$ est la loi de diffusion théorique donnée par:

$$
S^{t}(\vec{Q}, \omega)=A_{1}(\vec{Q}) \cdot R\left(Q_{0}, \omega\right)+\sum_{n=2}^{N} A_{n}(\vec{Q}) \cdot L_{n}(\omega) \otimes R(Q, \omega)
$$

où $A_{1}(\vec{Q})$ est l'EISF, les termes $A_{n}(\vec{Q})(n>1)$ sont les facteurs de structure inélastiques, $\left(a+b Q^{2}\right)$ est une ligne de base indépendante de l'énergie et $L_{n}(\omega)$ est une Lorentzienne de $1 / 2$ largeur à $1 / 2$ intensité (HWHM) $\tau_{\mathrm{n}}^{-1}$ :

$$
\mathrm{L}_{\mathrm{n}}(\omega)=\frac{1}{\pi} \frac{\tau_{\mathrm{n}}^{-1}}{\tau_{\mathrm{n}}^{-2}+\omega^{2}}
$$

Dans le cadre du modèle de rotation uniaxiale présenté dans la section précédente, la loi de diffusion $S^{t}(\vec{Q}, \omega)$ s'écrira:

$$
S^{t}(\vec{Q}, \omega)=\sum_{i=1}^{N} \sum_{j=1}^{N} \sum_{n=1}^{N} P\left(\phi_{i}\right) e^{i \vec{Q}\left(\vec{r}_{i}-\vec{r}_{j}\right)}\left[X_{j n}\right]\left[L_{n}(\omega)\right]\left[x_{j n}\right]^{-1}
$$

expression dans laquelle on reconnaît l'EISF lorsque $n=1\left(\tau_{1}^{-1}=0\right.$ et $\left.L_{1}(\omega)=\delta(\omega)\right)$.

La technique IQNS permet de sonder les mouvements sur une échelle de temps $10^{-9}-10^{-12} \mathrm{~s}$, selon le type de spectromètre utilisé. En règle générale, trois facteurs sont à considérer:

i - La résolution instrumentale,

ii - La fenêtre en énergie,

iii - La gamme en transfert de moment.

Les facteurs (i) et (ii) conditionnent la gamme des temps caractéristiques accessibles. Par exemple sur les spectromètres à très haute résolution (par exemple IN10 à l'I.L.L.) fonctionnant sur le principe de la rétro diffusion, la fenêtre en énergie est de l'ordre de $\pm 15 \mu \mathrm{eV}$ et la résolution (HWHM) de $0.5 \mu \mathrm{eV}$. Dans ces conditions, les mouvements détectables auront des temps caractéristiques $\tau$ dans la gamme $10^{-10}<\tau<10^{-}$ ${ }^{9} \mathrm{~s}$. Le gain en résolution se fait souvent au détriment de la gamme de transfert de moment. Par exemple 
sur un spectromètre à temps de vol comme IN5 (ILL), une résolution de $50 \mu \mathrm{eV}$ environ est obtenue avec un faisceau monochromatique de neutrons ayant une longueur d'onde de $5 \AA$. Dans ces conditions, la fenêtre en transfert de moment est $0<\mathrm{Q}<2.2 \AA^{-1}$. Avec une longueur d'onde de $12 \AA$, la résolution de l'appareil est de l'ordre de $10 \mu \mathrm{eV}$ mais avec $0<\mathrm{Q}<0.9 \AA^{-1}$. Il est donc souhaitable de réaliser des séries d'expériences dans des conditions différentes afin de bénéficier à la fois de spectres enregistrés à haute et moyenne résolution et accéder ainsi à des gammes temporelles et spatiales étendues.

L'analyse des spectres IQNS reposant sur des procédures d'ajustement, il est possible de réaliser une analyse qualitative des profils avec $N=1$ dans l'équation $(11), A_{1}(Q)$ et $\tau_{1}^{-1}\left(\tau_{1}^{-1}\right.$ étant dans ce cas fonction de $\mathrm{Q}$ ) sont les paramètres ajustables. Cette analyse permet d'estimer un EISF "effectif" (c'est à dire dépendant de la résolution instrumentale) et l'évolution des largeurs en fonction de $Q$.

\subsection{Spectroscopie $\mathrm{RMN}^{2} \mathrm{H}$}

Les noyaux ayant un spin nucléaire supérieur ou égal à 1 possèdent un moment électrique quadrupolaire (cas de l'atome de deutérium). Ce quadrupôle se couple (à la position du noyau ${ }^{2} \mathrm{H}$ ) avec le gradient de champ électrique $\overline{\bar{V}}$ qui résulte de l'asymétrie de la distribution des charges dues aux électrons et aux autres noyaux. Pour l'atome de deutérium le tenseur gradient de champ électrique (schématisé sur la figure 1) présente une symétrie axiale et s'écrit:

$$
\overline{\bar{V}}=\left[\begin{array}{ccc}
V_{x x} & 0 & 0 \\
0 & V_{y y} & 0 \\
0 & 0 & V_{z z}
\end{array}\right]
$$

dans le système d'axes principaux (celui de la figure 1), c'est à dire celui pour lequel la liaison $\mathrm{R}-\mathrm{D}(\mathrm{R}=$ $C, N, \ldots$ ) est l'axe $z$ (par convention $\left|V_{z z}\right| \geq\left|V_{y y}\right| \geq\left|V_{x x}\right|$ ). En tenant compte, en plus de l'effet Zeeman (figure 2), de l'interaction du gradient de champ électrique avec le moment quadrupolaire nucléaire les trois niveaux d'énergie pour un spin $=1$ seront:

$$
\left\{\begin{array}{c}
\mathrm{E}_{-1}=\gamma \hbar \mathrm{B}_{0}+\frac{\mathrm{h} \chi}{4}\left(\mathrm{P}_{2}(\cos \Theta)+\frac{\eta}{2} \sin ^{2} \Theta \cos 2 \Phi\right) \\
\mathrm{E}_{0}=-\frac{\mathrm{h} \chi}{2}\left(\mathrm{P}_{2}(\cos \Theta)+\frac{\eta}{2} \sin ^{2} \Theta \cos 2 \Phi\right) \\
\mathrm{E}_{+1}=-\gamma \hbar \mathrm{B}_{0}+\frac{\mathrm{h} \chi}{4}\left(\mathrm{P}_{2}(\cos \Theta)+\frac{\eta}{2} \sin ^{2} \Theta \cos 2 \Phi\right)
\end{array}\right.
$$

où $\gamma$ est le facteur gyromagnétique, $\mathrm{P}_{2}$ le second polynôme de Legendre $\left.\left(3 \cos ^{2} \theta-1\right) / 2\right)$;

$$
\chi=\frac{\mathrm{eQV}_{\mathrm{zz}}}{\mathrm{h}} \quad \eta=\frac{\left|\mathrm{V}_{\mathrm{yy}}\right|-\left|\mathrm{V}_{\mathrm{xx}}\right|}{\left|\mathrm{V}_{\mathrm{zz}}\right|}
$$

$\chi$ est la constante de couplage quadrupolaire "statique", $\eta$ est le paramètre d'asymétrie (grandeurs caractéristiques de la liaison $\mathrm{R}-\mathrm{D}$ ) et $\mathrm{eQ}$ est l'amplitude du moment électrique quadrupolaire. Dans ces expressions, les angles $\Theta$ et $\Phi$ représentent respectivement l'angle polaire et l'angle azimutal de l'axe $\mathrm{Oz}$ par rapport au référentiel du Laboratoire (OXYZ) dans lequel l'axe OZ est défini par le champ $\overrightarrow{\mathrm{B}}_{0}$ (figure 1). On attend donc un profil spectral constitué de raies aux fréquences $v_{ \pm 1}$ : 


$$
v_{ \pm 1}=v_{0} \pm \frac{3 \chi}{4} A \quad \text { avec } \quad A=P_{2}(\cos \Theta)+\frac{\eta}{2} \sin ^{2} \Theta \cos 2 \Phi
$$

où $v_{0}$ est la fréquence de Larmor $\left(\gamma \hbar \mathrm{B}_{0}\right)$. Pour l'atome de deutérium dans une liaison $\mathrm{C}-\mathrm{D}, \eta \approx 0$ de sorte que:

$$
\overline{\bar{V}}=\left[\begin{array}{ccc}
\mathrm{V}_{\mathrm{xx}} & 0 & 0 \\
0 & \mathrm{~V}_{\mathrm{yy}} & 0 \\
0 & 0 & \mathrm{~V}_{\mathrm{zz}}
\end{array}\right] \approx \frac{\mathrm{eq}}{2}\left[\begin{array}{ccc}
-1 & 0 & 0 \\
0 & -1 & 0 \\
0 & 0 & 2
\end{array}\right]
$$

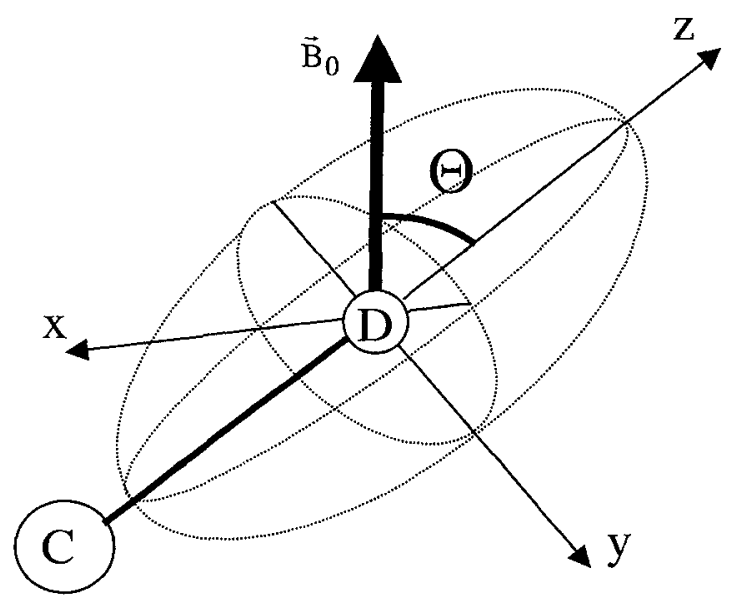

Figure 1: représentation schématique du tenseur gradient de champ électrique comme un ellipsoïde autour de l'atome de deutérium dans une liaison sigma C-D.

La spectroscopie $\mathrm{RMN}{ }^{2} \mathrm{H}$ sonde essentiellement l'orientation du tenseur gradient de champ électrique par rapport au champ d'induction magnétique statique extérieur $\overrightarrow{\mathrm{B}}_{0}$ qui provoque l'interaction Zeeman. Contrairement à la technique IQNS qui sonde des déplacements atomiques, la $\mathrm{RMN}{ }^{2} \mathbf{H}$ sondera les orientations des liaisons R-D par rapport au champ extérieur, que ces orientations évoluent ou non au cours du temps. Placés dans le champ $\overrightarrow{\mathrm{B}}_{0}$, les spins nucléaires vont tendre à s'aligner dans la direction du champ avec création d'une magnétisation résultante $\overrightarrow{\mathrm{M}}$. Un deuxième champ d'induction $\overrightarrow{\mathrm{B}}^{\prime}$ polarisé linéairement est alors appliqué perpendiculairement à $\overrightarrow{\mathrm{B}}_{0}$ de manière à écarter la magnétisation résultante de sa position d'équilibre. La détection étant réalisée dans le plan, on mesure la relaxation de la composante transverse de la magnétisation. Deux mécanismes de relaxation ont lieu que l'on peut détecter directement (relaxation transverse ou spin-spin dont le temps de caractéristique est noté $\mathrm{T}_{2}$ ) ou indirectement (relaxation longitudinale ou spin-réseau notée $T_{1}$ ). Le mécanisme $T_{2}$ est lié à la relaxation de la phase des différents moments angulaires de spin et le mécanisme $T_{1}$ au retour à l'équilibre de l'aimantation résultante $\left(\mathrm{T}_{2}<\mathrm{T}_{1}\right)$. La mesure de ces fonctions de relaxation est réalisée à l'aide de séquences d'impulsions spécifiques et nous allons voir que des informations sur la dynamique aux temps 
courts et aux temps longs peuvent être ainsi obtenues (pour plus de détails sur le fonctionnement d'un spectromètre RMN, consulter par exemple la référence [5]).

(b)

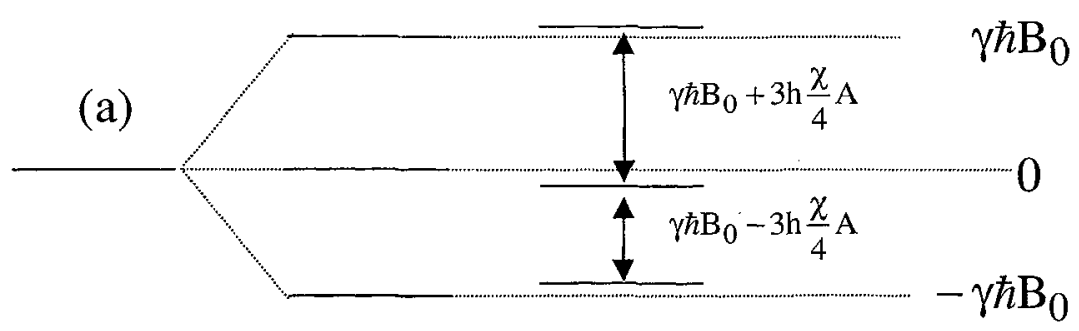

Figure 2: (a) noyau non perturbé, (b) éclatement Zeeman, (c) éclatement Zeeman perturbé par l'interaction du gradient de champ électrique avec le moment électrique quadrupolaire.

\subsubsection{Relaxation $T_{2}$ : profils spectraux.}

Le mécanisme de relaxation $T_{2}$ résulte du déphasage entre les spins nucléaires. En d'autres termes, la fréquence de résonance sera modulée par les mouvements de rotation. Supposons que les réorientations d'une liaison C-D $(\eta=0)$ dans le référentiel du laboratoire soient très lentes, c'est à dire "gelées". Dans ces conditions, la fréquence de résonance $v_{\mathrm{r}}$ est donnée par l'équation (17):

$$
\pm v_{\mathrm{r}}=v_{ \pm 1}-v_{0}= \pm \frac{3 \chi}{4} \mathrm{P}_{2}(\cos \Theta)
$$

Le spectre RMN pour une liaison C-D faisant un angle $\Theta$ avec le champ d'induction $\vec{B}_{0}$ présentera deux raies symétriquement disposées de part et d'autre de la fréquence de Larmor $v_{0}$. La composante transverse (notée $\vec{M}_{ \pm}$) de la magnétisation $\vec{M}(t)$ n'étant pas modulée par les réorientations, le mécanisme de déphasage cité ci-dessus est un processus lent par rapport à la fréquence de résonance $\pm v_{\mathrm{r}}=\left(v_{ \pm 1}-v_{0}\right)$ et nous aurons:

$$
\vec{M}_{ \pm}(t)=\vec{M}(0)\left(e^{i 2 \pi v_{r} t}+e^{-i 2 \pi v_{r} t}\right)
$$

La transformée de l'équation (20) donne bien le spectre attendu composé de deux raies $\delta\left(v \pm v_{\mathrm{r}}\right)$.

Considérons maintenant une liaison C-D animée d'un mouvement de rotation tel que l'angle $\Theta$ dépend du temps t. Dans ces conditions la précession du spin nucléaire dépend également du temps et la fréquence de résonance $v_{\mathrm{r}}$ est une variable stochastique:

$$
\vec{M}_{ \pm}(t)=\vec{M}_{ \pm}(0)\left(\left\langle\exp \left(i 2 \pi \int_{0}^{t} d t^{\prime} v_{r}\left(t^{\prime}\right)\right)\right\rangle+\left\langle\exp \left(-i 2 \pi \int_{0}^{t} d t^{\prime} v_{r}\left(t^{\prime}\right)\right)\right\rangle\right)
$$

On peut montrer [6] que l'équation ci-dessus peut également s'écrire, si on considère la méthode de discrétisation développée dans la section modèles, comme: 


$$
\left\langle\exp \left(i 2 \pi \int_{0}^{t} d t^{\prime} v_{r}\left(t^{\prime}\right)\right)\right\rangle=\sum_{n=1}^{N} P\left(\phi_{n}\right) \sum_{i=1}^{N} \sum_{j=1}^{N}\left[X_{j n}^{\prime}\right] e^{-\lambda_{n}^{\prime}|t|}\left[X_{n i}^{\prime}\right]^{-1}
$$

où les termes $\lambda_{n}^{\prime}$ sont les valeurs propres et les termes [X'] les vecteurs propres colonne de la matrice $\mathrm{i} 2 \pi\left\|v_{\mathrm{ij}}\right\|+\left\|\mathrm{L}_{\mathrm{ij}}\right\|$, la matrice $\left\|\mathrm{L}_{\mathrm{ij}}\right\|$ ayant été défini auparavant (équation (5)). La matrice des fréquences $\left\|v_{\mathrm{ij}}\right\|$ est diagonale, chaque terme $v_{\mathrm{ii}}$ (les termes $v_{\mathrm{ij}}$ sont nuls pour $\mathrm{i} \neq \mathrm{j}$ ) correspondant à la fréquence de résonance $v_{r}^{i}$ pour chacune des $N$ orientations $\Theta_{i}$ de la liaison C-D par rapport au champ extérieur. La transformée de Fourier de la fonction ci-dessus est le profil spectral attendu.

Dans la limite pour laquelle les réorientations sont très rapides, (les éléments de la matrice $\left\|\mathrm{L}_{\mathrm{ij}}\right\|$ sont grands devant $v_{r}$ ) seul le terme tel que $\lambda_{n}^{\prime}=v_{r}^{n}$ sera significatif (c'est à dire pour la valeur propre nulle de la matrice $\left\|\mathrm{L}_{\mathrm{ij}}\right\|$ ). Le spectre va donc se réduire à deux simples Lorentziennes centrées sur les fréquences de résonance moyennes

$$
\pm v_{\mathrm{r}}^{*}= \pm \sum_{\mathrm{i}=1}^{\mathrm{N}} \mathrm{P}\left(\phi_{\mathrm{i}}\right) v_{\mathrm{r}}^{\mathrm{i}}
$$

(4)

(3)

(2)

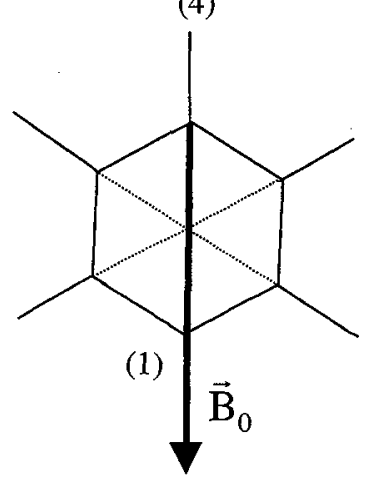

Figure 3: cas hypothétique d'une molécule de benzène $C_{6} D_{6}$ dont une liaison $C-D$ est parallèle au champ d'induction $B_{0}$.

Pour illustrer l'évolution du spectre entre les limites de modulations lente et rapide de la fréquence de résonance, nous avons choisi de considérer la situation hypothétique d'une molécule de benzène perdeutériée $\mathrm{C}_{6} \mathrm{D}_{6}$. Pour simplifier, nous supposerons q'une des liaisons $C-D$ (site $(1)$ ) fait un angle $\Theta_{1}=0$ par rapport au champ d'induction $\overrightarrow{\mathrm{B}}_{0}$ (figure 3). Dans la limite statique, deux liaisons $\mathrm{C}-\mathrm{D}$ sont parallèles à $B_{0}\left(\Theta_{1}=0\right.$ et $\left.\Theta_{4}=\pi\right)$, deux font un angle de $\pi / 3\left(\Theta_{2}\right.$ et $\left.\Theta_{6}\right)$ et deux de $2 \pi / 3\left(\Theta_{3}\right.$ et $\left.\Theta_{5}\right)$. Conformément à l'équation (19) on attend un spectre de quatre raies, deux aux fréquences $\pm v_{\mathrm{r}}= \pm 3 \chi / 4$ et deux à $\pm v_{\mathrm{r}}=\mp$ $3 \chi / 32$.

Supposons maintenant que la molécule de benzène effectue des réorientations par rapport à l'axe $\mathrm{C}_{6}$ de la molécule. Afin que la symétrie d'ordre six soit statistiquement conservée, nous adoptons un modèle de réorientations par sauts sur six orientations équiprobables (ce modèle est présenté dans l'article de $P$. Damay [4]). La fréquence de transition pour un saut de $2 \pi / 6$ est définie par le paramètre $\mathrm{k}$. Dans la limite 
des réorientations rapides par rapport à l'axe de symétrie $\mathrm{C}_{6}$ de la molécule de benzène on s'attend, en vertu de l'équation (23), à observer deux raies seulement à $\pm v^{*}{ }_{r}= \pm 3 \chi / 16$.

Nous avons reporté sur la figure 4 l'évolution du profil RMN en fonction de la fréquence de transition $\mathrm{k}$ pour le modèle de réorientations sur 6 sites. Nous observons dans un premier temps un élargissement des 4 raies puis le phénomène de coalescence conduisant au profil constitué de deux raies seulement. Les largeurs de ces deux raies n'évoluent plus lorsque $\mathrm{k}>10^{7} \mathrm{~s}^{-1}$. Nous voyons bien que l'étude de ces profils nous permet de caractériser la dynamique réorientationnelle de la molécule de benzène lorsque $\mathrm{k}<10^{7} \mathrm{~s}^{-1}$. Pour comparaison le profil de diffusion IQNS est constitué d'un pic élastique et de trois Lorentziennes (équations (11-13) avec $\mathrm{N}=3$ ) de largeurs $\mathrm{k}, 3 \mathrm{k}$ et $4 \mathrm{k}$. Il faudrait donc disposer d'un spectromètre dont la résolution est au moins de l'ordre de $10^{-2} \mu \mathrm{eV}$ pour sonder ces mouvements sur une même échelle de temps que la RMN.

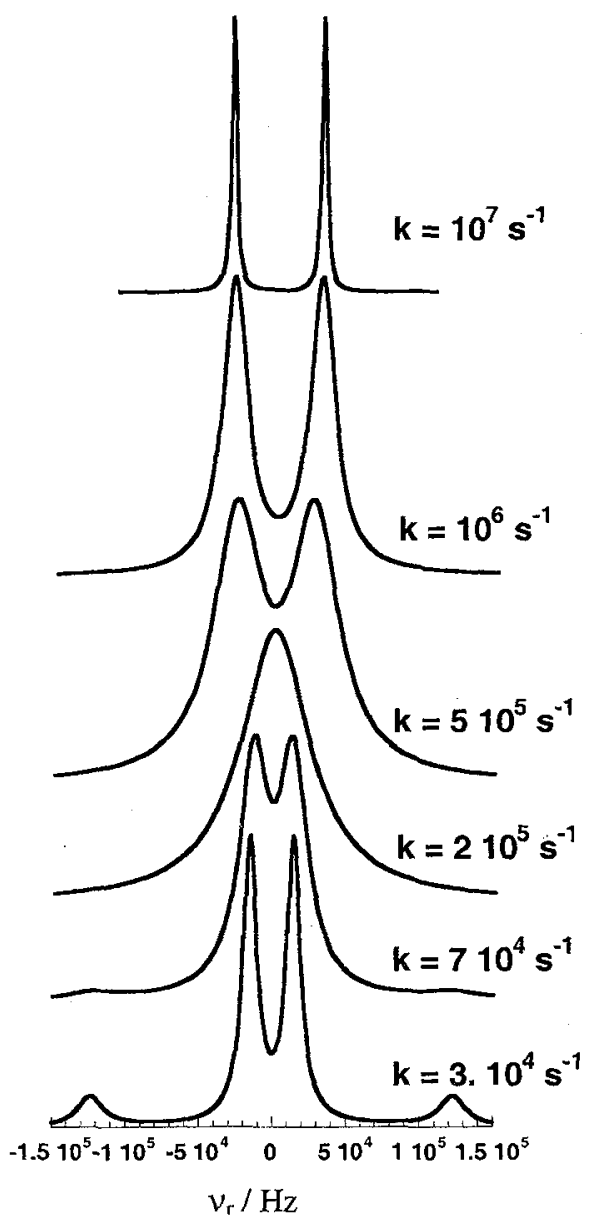

Figure 4: profils spectraux d'une molécule de benzène $\mathrm{C}_{6} \mathrm{D}_{6}$ effectuant des rotations par sauts par rapport à son axe de symétrie $\mathrm{C}_{6}$ perpendiculaire à l'induction $\mathrm{B}_{0}$. Ces profils ont été simulés avec un temps d'écho $\tau_{\mathrm{D}}=13 \mu \mathrm{s}$ et $\chi=163 \mathrm{kHz}$.

Nous avons vu dans l'exemple ci-dessus que la fréquence de résonance $v_{\mathrm{r}}^{*}$ pour une liaison C-D animée de mouvements rapides pouvait se déduire des fréquences $v_{\mathrm{r}}$ dans la limite statique qui dépendent des orientations $\Theta_{1}$ des liaisons $C-D$ par rapport au champ d'induction $B_{0}\left(B_{0}\right.$ perpendiculaire à l'axe $C_{6}$ de la molécule). Pour le modèle de rotations sur 6 sites équiprobables, le spectre résultant dans la limite 
de modulation rapide est indépendant de la valeur de l'angle $\Theta_{1}\left(\Theta_{i}=\Theta_{1}+(i-1) \pi / 3\right)$. Nous verrons par la suite que cette situation se résume à l'équation:

$$
\pm v_{\mathrm{r}}^{*}= \pm \frac{3 \chi^{*}}{4} P_{2}\left(\cos \Theta^{\prime}\right)
$$

où $\chi^{*}=\chi / 2$ et $\Theta^{\prime}$ est l'angle entre l'induction $\mathrm{B}_{0}$ et l'axe $\mathrm{C}_{6}$ de rotation. Pour des réorientations par sauts sur des sites équidistants mais non équiprobables, $v^{*}$ dépendra de $\Theta_{1}$. On peut donc obtenir des informations sur les aspects géométriques de la dynamique même si les temps caractéristiques du mouvement sont trop courts par rapport à la fenêtre temporelle de la technique.

Introduisons maintenant le concept de tenseur gradient de champ électrique "moyennisé" qui est d'une grande utilité lorsqu'on ne dispose pas d'échantillon monocristallin, mais d'une poudre. Le tenseur gradient de champ électrique $\overline{\bar{V}}$ du noyau de deutérium dans la liaison C-D s'écrit, dans le référentiel arbitraire du cristal ou de la molécule (figure 5), selon:

$$
\overline{\bar{V}}=-\frac{e q}{2}\left[\begin{array}{ccc}
1-3 \cos ^{2} \phi \sin ^{2} \theta & \frac{3}{2} \sin 2 \phi \sin ^{2} \theta & \frac{3}{2} \cos \phi \sin 2 \theta \\
\frac{3}{2} \sin 2 \phi \sin ^{2} \theta & 1-3 \sin ^{2} \phi \sin ^{2} \theta & -\frac{3}{2} \sin \phi \sin 2 \theta \\
\frac{3}{2} \cos \phi \sin 2 \theta & -\frac{3}{2} \sin \phi \sin 2 \theta & 1-3 \cos ^{2} \theta
\end{array}\right]
$$

Notons qu'après diagonalisation de ce tenseur on retrouve son expression (18) dans le système d'axes principaux. Considérons à nouveau le modèle de réorientations par sauts de la molécule par rapport à l'axe moléculaire $C_{6}$. Dans ces conditions l'angle $\theta$ est constant (figure 5) et le tenseur moyen $\overline{\bar{V}} *$ s'écrira:

$$
\overline{\mathrm{V}}^{*}=-\frac{\mathrm{eq}}{2}\left[\begin{array}{ccc}
1-3\left\langle\cos ^{2} \phi\right\rangle \sin ^{2} \theta & \frac{3}{2}\langle\sin 2 \phi\rangle \sin ^{2} \theta & \frac{3}{2}\langle\cos \phi\rangle \sin 2 \theta \\
\frac{3}{2}\langle\sin 2 \phi\rangle \sin ^{2} \theta & 1-3\left\langle\sin ^{2} \phi\right\rangle \sin ^{2} \theta & -\frac{3}{2}\langle\sin \phi\rangle \sin 2 \theta \\
\frac{3}{2}\langle\cos \phi\rangle \sin 2 \theta & -\frac{3}{2}\langle\sin \phi\rangle \sin 2 \theta & 1-3 \cos ^{2} \theta
\end{array}\right]
$$

En d'autres termes on trouve, après diagonalisation de (25), une expression analogue à l'équation (17):

$$
v_{ \pm 1}=v_{0} \pm \frac{3 \chi^{*}}{4}\left(P_{2}\left(\cos \Theta^{\prime}\right)+\frac{\eta^{*}}{2} \sin ^{2} \Theta^{\prime} \cos 2 \Phi\right)
$$

où, selon les conventions de définition du système d'axes principaux:

$$
\left|\mathrm{V}_{\mathrm{zz}}^{*}\right| \geq\left|\mathrm{V}_{\mathrm{yy}}^{*}\right| \geq\left|\mathrm{V}_{\mathrm{xx}}^{*}\right|, \chi^{*}=\frac{\mathrm{eQV}_{\mathrm{zz}}^{*}}{\mathrm{~h}} \text { et } \eta^{*}=\frac{\left|\mathrm{V}_{\mathrm{yy}}^{*}\right|-\left|\mathrm{V}_{\mathrm{xx}}^{*}\right|}{\left|\mathrm{V}_{\mathrm{zz}}^{*}\right|}
$$

et l'angle $\Theta^{\prime}$ représente l'angle que font l'axe $\mathrm{z}$ du système d'axes principaux et le champ d'induction $\mathrm{B}_{0}$ (figure 5). Plus précisément pour le modèle considéré plus haut $\theta=\pi / 2,\langle\sin \phi\rangle$ et $\langle\sin 2 \phi\rangle=0,\langle\cos \phi\rangle=$ 0 et $\left\langle\cos ^{2} \phi\right\rangle=1 / 2$. Dans ces conditions: 


$$
\overline{\bar{V}^{*}}=-\frac{\text { eq }}{2}\left[\begin{array}{ccc}
-1 / 2 & 0 & 0 \\
0 & -1 / 2 & 0 \\
0 & 0 & 1
\end{array}\right]
$$

soient $\chi^{*}=\chi / 2$ et $\eta^{*}=0$. Pour ce modèle l'axe $\mathrm{z}$ du système d'axes principaux est colinéaire à l'axe de rotation $\mathrm{Z}$ et $\Theta^{\prime}=\pi / 2$. On trouve alors $v_{\mathrm{r}}^{*}=3 \chi / 16$. Pour des sites non équiprobables, le paramètre d'asymétrie $\eta^{*}$ est non nul et la fréquence $v^{*}{ }_{r}$ dépend de $\Phi$ et de $\Theta^{\prime}$.

Cette discussion se réfère au cas hypothétique d'une molécule placée dans un champ d'induction $\mathrm{B}_{0}$ et peut donc s'appliquer, dans son principe, à l'étude de monocristaux. Pour une poudre, il convient d'intégrer numériquement la transformée de Fourier de l'équation (22) par rapport à toutes les orientations de $\mathrm{B}_{0}$ dans le référentiel moléculaire (ou cristallin). On peut cependant, dans les cas limites de modulation lente ou de modulation rapide, prévoir qualitativement le profil du spectre de poudre. Nous avons vu (équation (19)) que dans la limite statique, la fréquence de résonance dépendait de l'angle $\Theta$ que font une liaison C-D et le champ $B_{0}$. Pour une poudre, il conviendra alors de construire un spectre résultant de toutes les orientations (une sphère) que peut prendre la liaison $C-D$ par rapport à $B_{0}$. Il est facile de réaliser que des discontinuités dans le profil sont attendues lorsque $\Theta=0$ ou $\pi / 2$, c'est à dire lorsque la pente du second polynôme de Legendre $P_{2}(\cos \Theta)$ est nulle. Dans ces conditions, le spectre de poudre de la molécule de benzène dans la limite statique présentera quatre maxima à $\pm v_{\mathrm{r}}= \pm 3 \chi / 4$ et $\pm 3 \chi / 8$. Dans la limite de modulation rapide, il convient de considérer l'équation (24). En faisant le même raisonnement que ci-dessus, le modèle de sauts sur 6 orientations équiprobables prédit un spectre de poudre présentant également 4 maxima à $v_{\mathrm{r}}^{*}= \pm 3 \chi / 8$ et $\pm 3 \chi / 16$, soient des éclatements quadrupolaires diminués d'un facteur 2 par rapport à ceux du spectre de poudre dans la limite statique. Dans le régime intermédiaire, le spectre subira une évolution complexe entre ces deux profils.

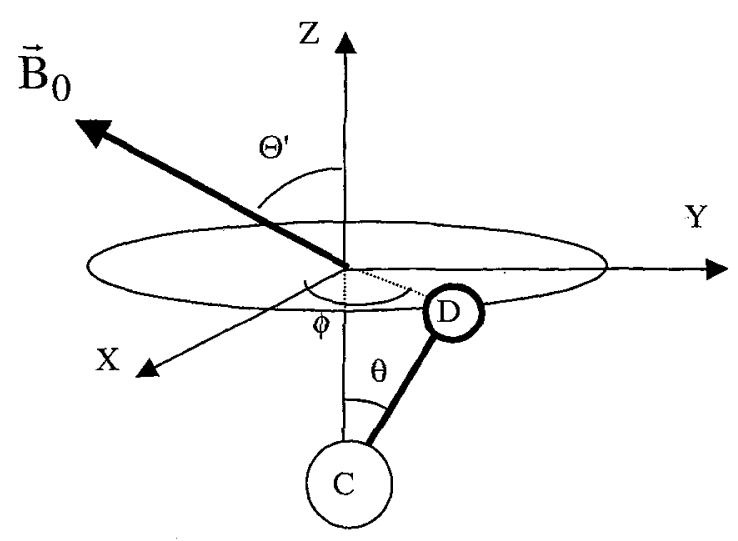

Figure 5: définition des orientations de la liaison C-D et du champ d'induction $\mathrm{B}_{0}$ dans un système d'axes cristallin ou moléculaire (ici l'axe $Z$ de rotation est colinéaire à l'axe $\mathrm{z}$ du système d'axes principaux lorsque $\eta^{*}=0$ ).

Considérons maintenant le modèle plus complexe de réorientations sur 6 sites équidistants mais non équiprobables. Supposons par exemple un site de probabilité $p_{1}$ et les 5 autres sites de probabilités égales à $\left(1-p_{1}\right) / 5$, le site de probabilité $p_{1}$ se situe sur l'axe des $\mathrm{x}(\phi=0)$. On trouve facilement avec $\theta=\pi / 2$ que $<\cos ^{2} \phi>=\left(2+3 p_{1}\right) / 5$ et $\left\langle\sin (2 \phi)>=0\right.$. Le tenseur $\overline{\bar{V}} *$ est diagonal et par exemple avec $\mathrm{p}_{1}=0,4, \chi^{*}=\chi / 2$ et $\eta^{*}=0,54$. Le spectre de poudre présentera alors six discontinuités, deux pour $\Theta^{\prime}=0$ aux fréquences de résonance $v_{\mathrm{r}}^{*}= \pm 3 \chi / 8$ et quatre autres $\left(\Theta^{\prime}=\pi / 2\right)$ aux fréquences $\pm 3 \chi\left(1 \pm \eta^{*}\right) / 16$. 


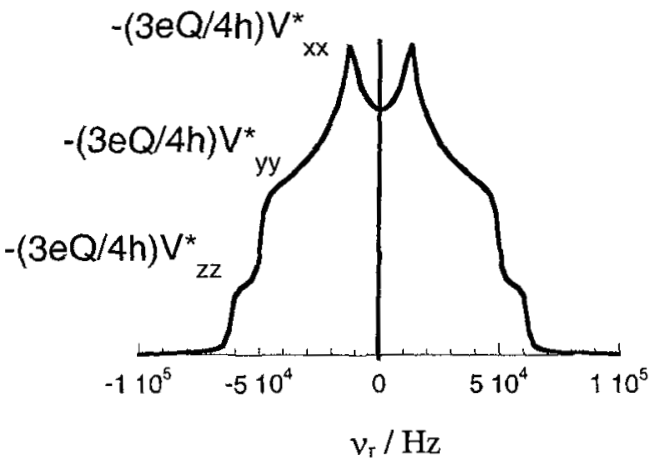

Figure 6: spectre de poudre présentant 6 discontinuités dans son profil. Dans la limite de modulation rapide, celles-ci nous permettent de déterminer les composantes du tenseur gradient de champ électrique "moyennisé" exprimé dans le système d'axes principaux.

Plus généralement, le simple examen du spectre de poudre nous permettra de déterminer les composantes du tenseur gradient de champ électrique moyennisé (figure 6). La dépendance du profil en fonction du paramètre effectif $\eta^{*}\left(\chi^{*}\right.$ constant) est reportée sur la figure 7 .

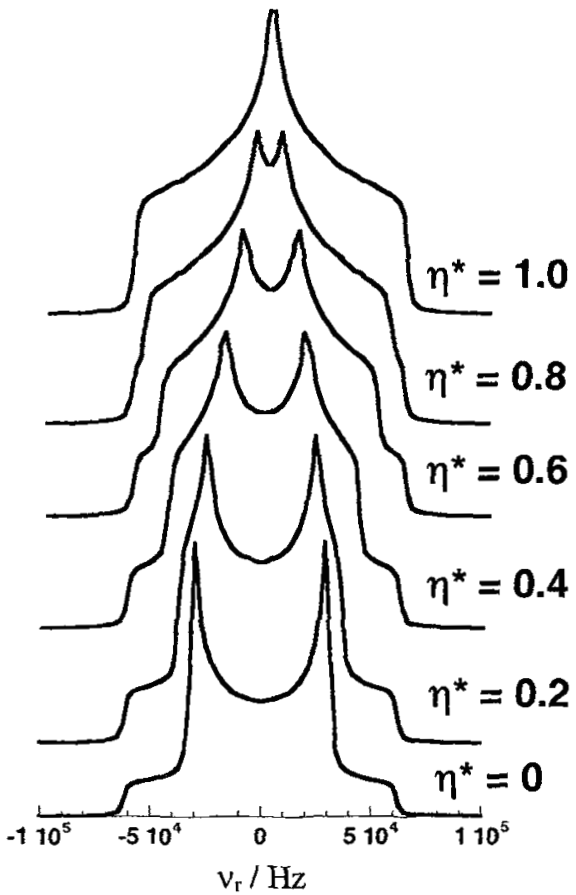

Figure 7: évolution des profils pour une poudre en fonction du paramètre d'asymétrie $\eta^{*}, \chi^{*}=\chi / 2(\chi=163 \mathrm{kHz})$.

\subsubsection{Relaxation $T_{l}$}

L'expression du temps de relaxation $T_{1}$ de la composante longitudinale de la magnétisation est de la forme [7]: 


$$
\frac{1}{\mathrm{~T}_{1}}=\frac{3 \pi^{2}}{4} \chi^{2}\left[\mathrm{~J}_{1}(\omega)+4 \mathrm{~J}_{2}(2 \omega)\right]
$$

où les termes $J_{m}(m \omega)(m=1,2)$ sont les densités spectrales, transformées de Fourier des fonctions de corrélation

$$
C_{m}(t)=\left\langle R_{2 m}(0) R_{2 m}(t)\right\rangle / \chi^{2}
$$

$R_{2 m}(t)$ est fonction des composantes du tenseur gradient de champ électrique dans le système d'axes principaux exprimé en coordonnées sphériques [7]. En général les expressions des densités spectrales $\mathrm{J}_{\mathrm{m}}(\omega)$ mettent en jeu une somme de termes $\mathrm{g}\left(\tau_{\mathrm{i}}, \mathrm{m} \omega\right)$ tels que:

$$
g\left(\tau_{i}, m \omega\right)=\frac{\tau_{i}}{1+m^{2} \omega^{2} \tau_{i}^{2}}
$$

où $\tau_{\mathrm{i}}$ est un temps caractéristique du mouvement, $\omega$ est la pulsation de Larmor pour le noyau de deutérium. Afin d'évaluer approximativement une échelle de temps caractéristiques, supposons que la relaxation du $T_{1}$ s'exprime à l'aide d'une simple Lorentzienne $(\mathrm{m}=1$ et $\mathrm{i}=1$ dans l'équation (32)). Dans ces conditions, la courbe représentant l'évolution de $T_{1}$ en fonction de $\tau_{1}$ présente un minimum lorsque $\omega \tau_{1}=1$. Par exemple, pour $\omega=310^{8} \mathrm{rds} \cdot \mathrm{s}^{-1}, \tau_{1}=310^{-7} \mathrm{~s}$. En d'autres termes, lorsqu'on réalise une mesure du $T_{1}$ en fonction de la température et que l'on observe un minimum $T_{1}{ }^{\text {min }}$ à la température $T_{0}$, on saura que qualitativement le temps caractéristique du mouvement sera plus petit $310^{-7} \mathrm{~s}$ pour des températures $\mathrm{T}>\mathrm{T}_{0}$ et supérieur à $310^{-7} \mathrm{~s}$ pour $\mathrm{T}<\mathrm{T}_{0}$.

Illustrons ces mesures de $T_{1}$ à l'aide du modèle de rotations par sauts sur 6 sites équidistants et équiprobables, sans présenter les calculs que l'on peut trouver par ailleurs [7]. L'évolution du $T_{1}$ en fonction de la constante de saut $\mathrm{k}$ est présentée sur la figure 8 . Cette situation correspond à l'exemple de la figure 3 où le champ magnétique extérieur fait un angle de $\pi / 2$ par rapport à l'axe de rotation $\mathrm{C}_{6}$ de la molécule de benzène. Le $\mathrm{T}_{1}$ pour ce modèle présente un minimum pour $\mathrm{k}=10^{8} \mathrm{~s}^{-1}$, ce qui correspond à la limite de modulation rapide pour les profils spectraux. En principe donc, les mesures combinées des profils de relaxation $\left(\mathrm{T}_{2}\right)$ et des temps $\left(\mathrm{T}_{1}\right)$ devraient permettre de sonder les mouvements sur une échelle de temps très étendue. Il est d'usage d'élaborer un modèle dynamique à partir des profils spectraux et l'analyse des temps $T_{1}$ permet de préciser les temps caractéristiques du mouvement.

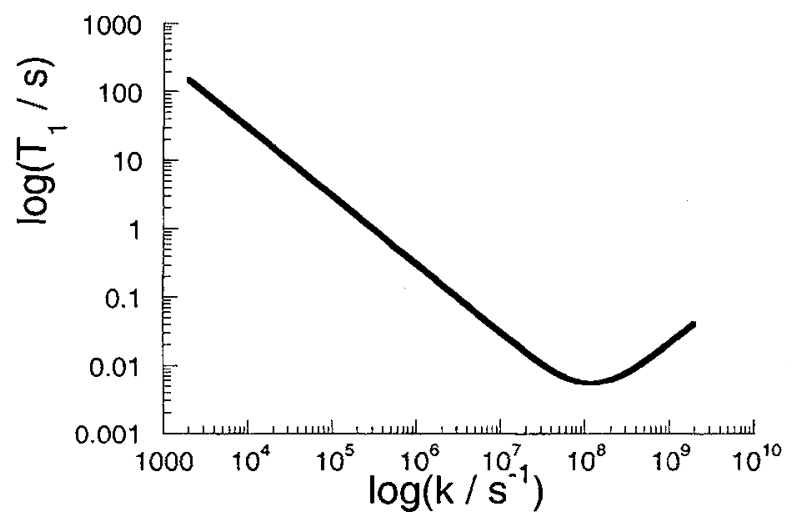

Figure 8: variation du $T_{1}$ pour le modèle de rotations par sauts sur 6 sites en fonction de la constante de saut $k$. 


\subsection{Simulations MD}

Les simulations numériques de la dynamique moléculaire (MD) vont nous fournir une aide précieuse pour l'analyse des données expérimentales. Rappelons en effet que le principe d'une simulation MD consiste, à partir d'un champ de forces, à déterminer des trajectoires atomiques [8]. En général les temps de simulation peuvent parfois atteindre la nanoseconde, mais rarement la microseconde. Il en résulte que compte tenu de ces échelles de temps, une comparaison directe avec les expériences IQNS est naturelle et peut répondre à aux moins deux objectifs:

- d'une part confronter le modèle utilisé pour simuler la dynamique moléculaire (boîte primaire, champ de force) à l'expérience,

- d'autre part permettre de tester la validité de la méthode employée pour analyser les données expérimentales.

Des spectres IQNS "simulés" peuvent être calculés à partir des trajectoires atomiques et comparés à l'expérience permettant dans un premier temps de "valider" la qualité du modèle utilisé dans la simulation MD. Dans une seconde étape on peut construire des modèles théoriques simples et tester la validité des approximations faites par comparaison avec les simulations. Par exemple des fonctions de distribution des orientations $\mathrm{P}(\phi)$ (équation (3)) sont extraites à partir des trajectoires atomiques et permettent d'élaborer un modèle sur la base de l'équation de Fokker-Planck (équations (1-2)). Nous illustrerons à l'aide d'un exemple (\$4.2) l'apport des simulations numériques pour l'analyse des données expérimentales.

\subsection{Discussion}

Nous venons de voir que les deux techniques, IQNS et $\mathrm{RMN}{ }^{2} \mathrm{H}$, permettent à l'expérimentateur d'obtenir des informations précises sur les aspects géométriques et dynamiques des fluctuations Browniennes de réorientation d'une molécule organique. Ces informations sont toutefois complémentaires. Nous avons souligné qu'une des limitations majeures de la spectroscopie IQNS est liée à la résolution instrumentale qui ne permet pas de sonder les mouvements sur des temps plus longs que $10^{9} \mathrm{~s}$ environ. De même une des limitations majeures de la spectroscopie $\mathrm{RMN}{ }^{2} \mathrm{H}$ est son incapacité à apporter des informations précises sur les temps caractéristiques de la dynamique aux temps courts $\left(<10^{7} \mathrm{~s}\right)$. Ces différences de sensibilité justifient pleinement le terme de complémentarité lorsqu'on compare ces deux techniques.

D'autres aspects importants méritent d'être soulignés. En tout premier lieu, l'expérimentateur désirant réaliser des spectres IQNS se trouve confronter au choix d'un spectromètre approprié en termes de résolution instrumentale, de fenêtre en énergie et de gamme de transfert de moment. Il est donc parfois indispensable de faire appel à la RMN (du deutérium ou du proton) afin de réaliser une estimation préliminaire de l'échelle des temps caractéristiques du mouvement. En effet, rappelons que la simple mesure sur une poudre de la variation du temps de relaxation $T_{1}$ en fonction de la température nous permet d'une part de déterminer à partir de quelle température les mouvements sont suffisamment rapides pour pouvoir être détectés en IQNS (c'est à dire pour des températures $T>T_{0}$ ). De plus, la variation de $\ln \left(\mathrm{T}_{1}\right)$ en fonction de $1 / \mathrm{T}$ vont nous permettre de déterminer une énergie d'activation et donc d'estimer le temps caractéristique du mouvement quelle que soit la température $\mathrm{T}$ pour $\mathrm{T}>\mathrm{T}_{0}$.

En IQNS, deux fonctions caractéristiques sont généralement analysées, l'EISF et la largeur du profil $\mathrm{S}(\mathrm{Q}, \omega)$. A condition d'opérer dans des conditions de résolution instrumentale satisfaisantes, il est possible de déterminer un EISF expérimental nous permettant en particulier d'obtenir des informations sur l'amplitude des réorientations (ou des translations). Aux temps courts, le profil du spectre $\mathrm{RMN}{ }^{2} \mathrm{H}$ nous permettra de préciser l'existence d'un axe de symétrie statistique. Nous verrons que pour des solides cristallins, ces deux informations sont complémentaires et nous permettent de lever nombre des ambiguittés propres à chacune de ces deux techniques prises indépendamment.

Pour conclure, précisons que seule la $R M N^{2} \mathrm{H}$ est adaptée pour l'étude de la dynamique moléculaire aux temps longs, c'est à dire pour des temps caractéristiques du mouvement supérieurs à $10^{-7} \mathrm{~s}$. Rappelons 
en effet qu'aux temps longs, des informations relativement précises à la fois sur la géométrie des mouvements et sur leur dynamique est obtenue en combinant des mesures de profils spectraux et de temps de relaxation $T_{1}$.

\section{EXEMPLES}

\subsection{Le benzène chrome tricarbonyle $\left(\eta^{6}-\mathrm{C}_{6} \mathrm{D}_{6}\right) \mathrm{Cr}(\mathrm{CO})_{3}$}

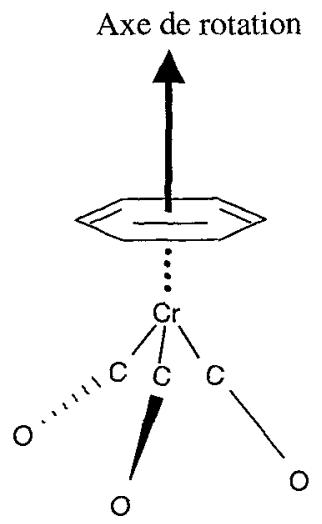

Figure 9: la molécule de BCTC.

Le benzène chrome tricarbonyle $\eta^{6}-\mathrm{C}_{6} \mathrm{H}_{6} \mathrm{Cr}(\mathrm{CO})_{3}$ (noté $\mathrm{BCTC}-\mathrm{h}_{6}$ ) est un complexe organo-métallique (figure 9) cristallisant dans une structure monoclinique. Aucune transition de phase structurale n'est observée entre 10 et $300 \mathrm{~K}$ mais les molécules de BCTC sont animées de mouvements de réorientation.

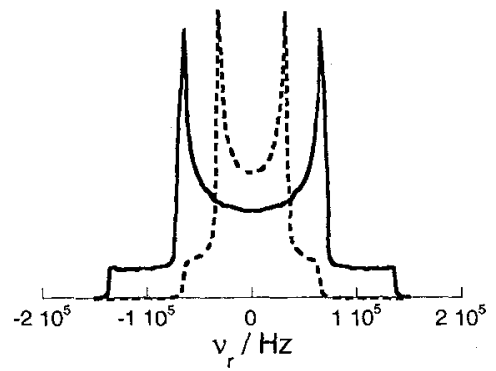

Figure 10: spectres ${ }^{2} \mathrm{H}$ RMN sur poudre du cristal de BCTC à $90 \mathrm{~K}$ (trait plein) et $300 \mathrm{~K}$ (trait pointillé).

Le spectre $\mathrm{RMN}{ }^{2} \mathrm{H}$ [9] enregistré à basse température sur de la poudre du composé deutérié $\eta^{6}$ $\mathrm{C}_{6} \mathrm{D}_{6} \mathrm{Cr}(\mathrm{CO})_{3}$ (noté $\mathrm{BCTC}-\mathrm{d}_{6}$ ) est reproduit sur la figure 11. Le profil correspond à la limite "statique" et peut être simulé parfaitement avec $\chi=183 \mathrm{kHz}$ et $\eta=0.05$ (nous poserons $\eta=0$ par la suite). A température ambiante, le profil observé montre que $\chi^{*}=\chi / 2$ et $\eta^{*}=0$ (figure 10). Dans ces conditions, la modulation est rapide et l'axe de rotation est un axe de symétrie. 


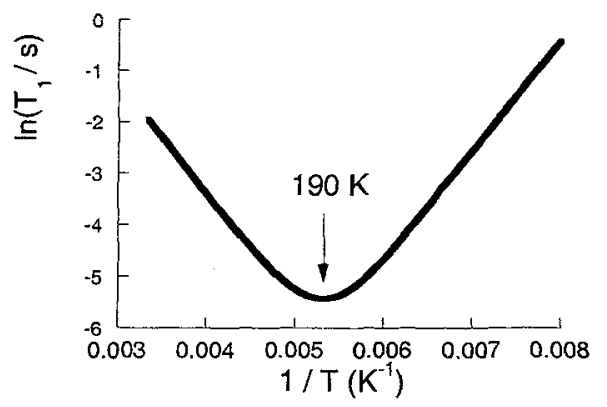

Figure 11: temps de relaxation $T_{1}$ expérimental pour la poudre de BCTC-d ${ }_{6}$.

Les mesures du temps $T_{1}$ en fonction de la température montrent que $T_{0}=190 \mathrm{~K}$ (figure 11). On en conclut donc que la dynamique de rotation est rapide pour $\mathrm{T}>190 \mathrm{~K}$ et dans un régime intermédiaire pour $\mathrm{T}<190 \mathrm{~K}$. Plus précisément les variations des profils spectraux entre $119 \mathrm{~K}$ et $160 \mathrm{~K}$ (figure 12 ) traduisent donc la dynamique réorientationnelle du BCTC- $d_{6}$ aux temps longs $\left(>10^{-7} \mathrm{~s}\right)$. Un modèle probable pour la partie benzénique $\mathrm{du} B C T C-\mathrm{d}_{6}$ correspond à des réorientations reproduisant soit un axe de symétrie d'ordre trois soit un axe de symétrie d'ordre 6 (figure 9).

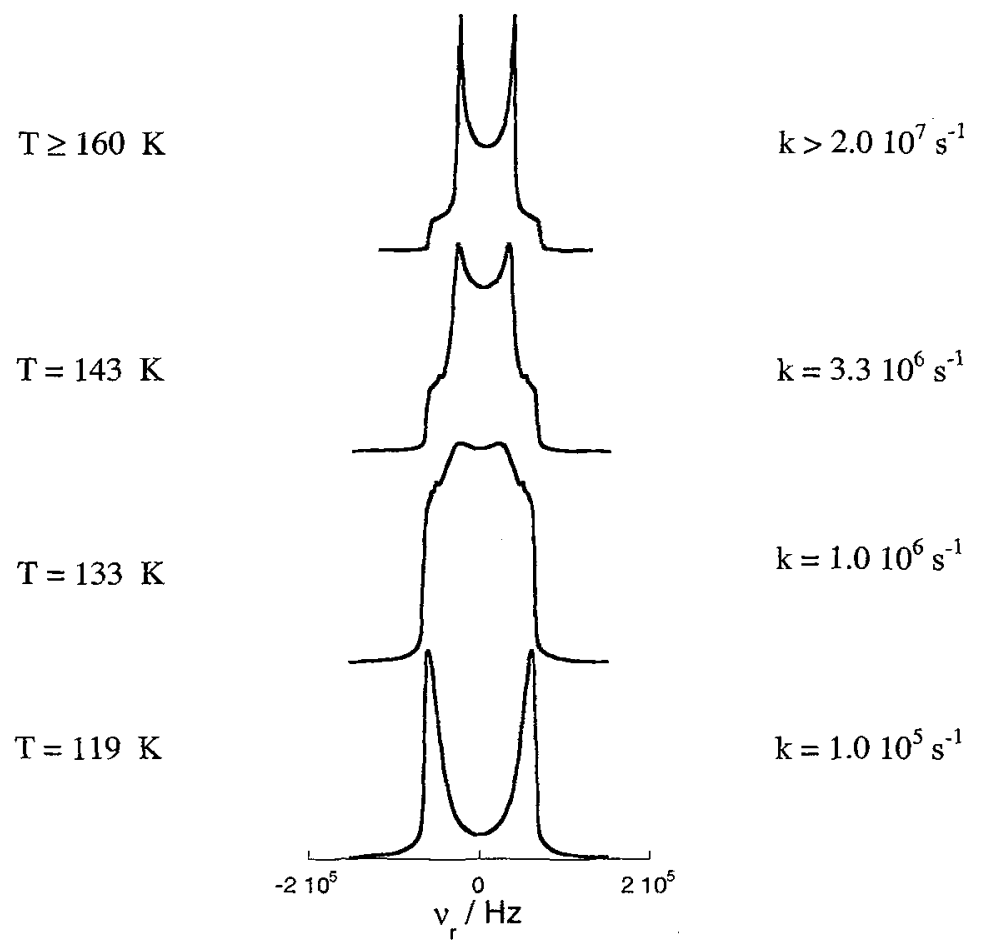

Figure 12: évolution en fonction de la température des spectres ${ }^{2} \mathrm{H}$ RMN de poudre du cristal de BCTC-d 6 : A droite les valeurs des paramètres $\mathrm{k}$ obtenus par ajustement des profils pour un modèle de réorientations par sauts sur 6 sites.

A partir de l'analyse qualitative des profils RMN, on peut conclure que la moitié benzénique du BCTC- $\mathrm{d}_{6}$ est animée de mouvements de rotation par rapport à un axe de symétrie d'ordre supérieur ou 
égal à trois (car $\chi^{*}=\chi / 2$ et $\left.\eta^{*}=0\right)$ et que les temps caractéristiques des mouvements sont supérieurs à environ $10^{-7}$ s pour $\mathrm{T}<160 \mathrm{~K}$.

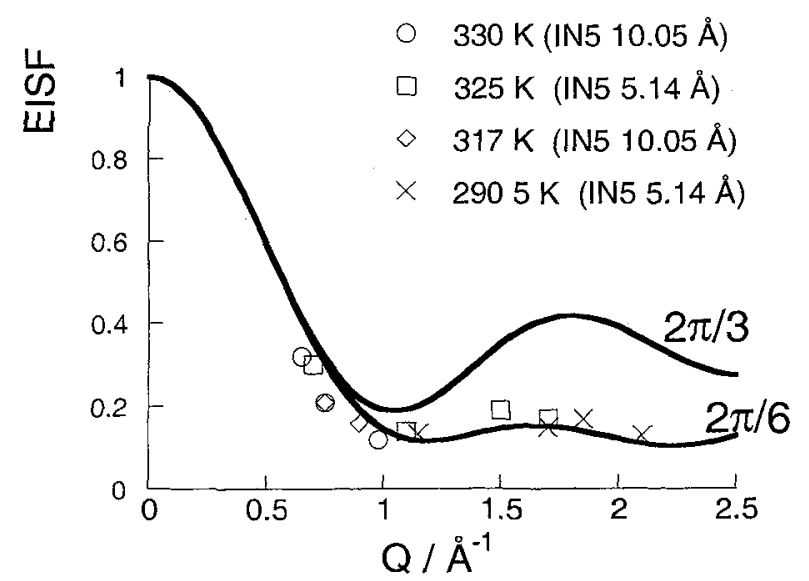

Figure 13: comparaison des EISFs "expérimentaux" et théoriques (sauts sur 3 et 6 sites) pour la poudre du cristal BCTC-h, Pour les fonctions théoriques, le calcul a été réalisé avec un rayon de giration du benzène de $2.48 \AA$.

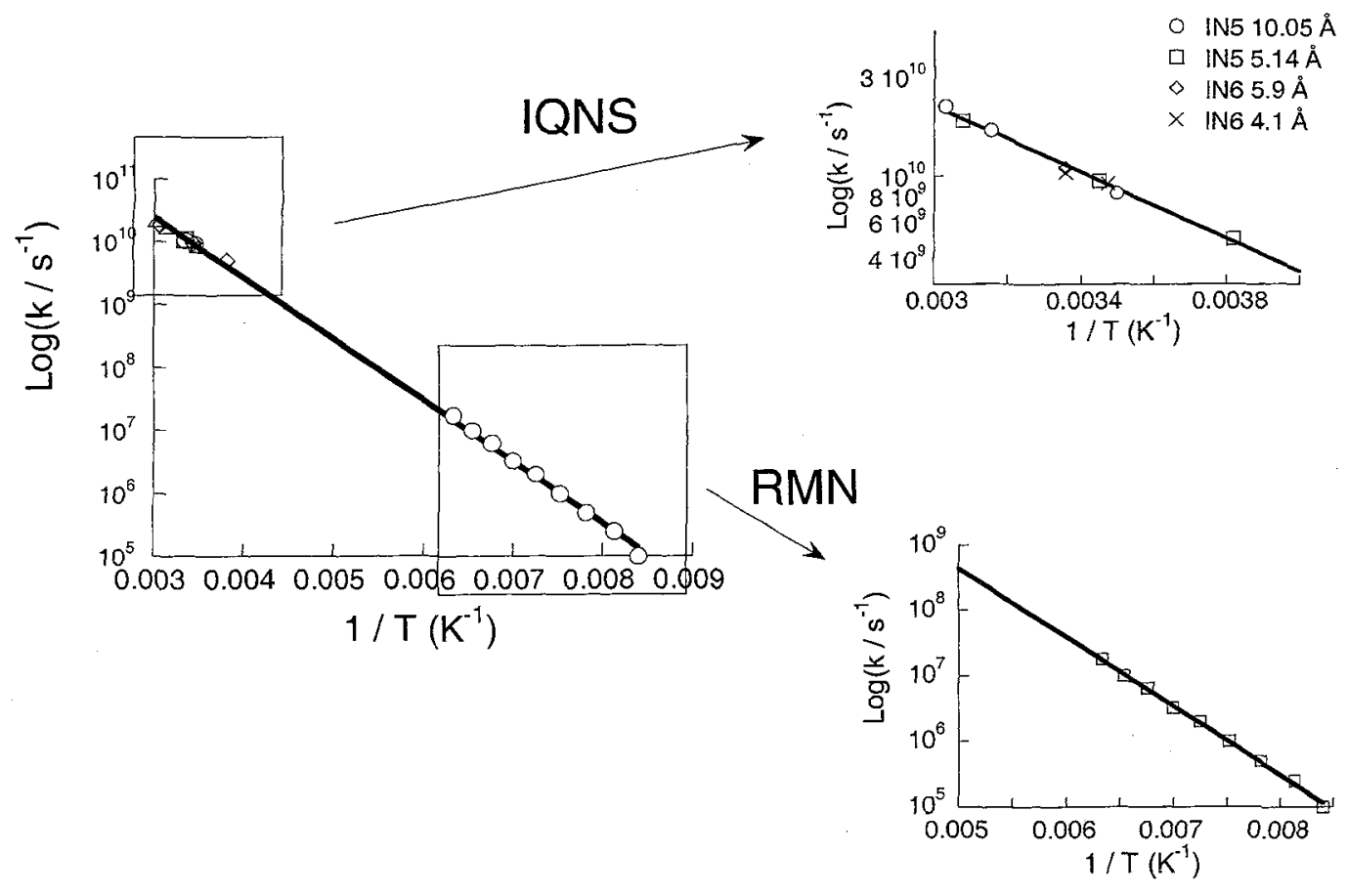

Figure 14: variations des constantes de sauts extraites de l'analyse des profils spectraux IQNS et ${ }^{2} \mathrm{H}$ RMN en fonction de la température. Les courbes en traits pleins représentent l'ajustement de fonctions d'Arrhenius sur les données expérimentales.

Les spectres IQNS du BCTC-h $h_{6}$ ont été obtenus sur les instruments IN5 et IN6 à l'ILL [10]. L'utilisation de ces spectromètres répond au besoin d'analyser les mouvements du BCTC sur des fenêtres 
temporelles et spatiales étendues. Ainsi les résolutions instrumentales varient de $10 \mu \mathrm{eV}$ (IN5 avec $\lambda_{0}=$ $10 \AA$ ) à $85 \mu \mathrm{eV}$ (IN6 avec $\lambda_{0}=4.1 \AA$ ) et les gammes de transfert de moment correspondantes sont respectivement $\left(0.2-1.1 \AA^{-1}\right)$ et $\left(0.3-2.6 \AA^{-1}\right)$. Une première estimation qualitative des profils de diffusion IQNS révèle que pour des températures inférieures à $200 \mathrm{~K}$, l'intensité est entièrement élastique. En d'autres termes, la dynamique de réorientation de la moitié benzénique du BCTC- $\mathrm{h}_{6}$ est lente par rapport à la résolution IQNS pour $\mathrm{T}<200 \mathrm{~K}$.

Une estimation des EISFs pour des températures supérieures à $290 \mathrm{~K}$ (à des températures pour lesquelles les élargissements des profils sont clairement résolus) a été réalisée permettant ainsi la comparaison avec les EISFs théoriques pour des modèles de rotations par sauts sur 3 et 6 sites (figure 13). Cette comparaison illustre remarquablement bien le fait que, contrairement à la $R M N{ }^{2} \mathrm{H}$, la technique IQNS permet de discriminer entre les modèles de rotation sur 3 ou 6 sites.

Nous pouvons donc conclure qu'un modèle de rotations par sauts instantanés sur 6 sites de la moitié benzénique du BCTC est convenable. On peut aller plus loin dans l'analyse en ajustant les profils théoriques aux spectres expérimentaux (IQNS et $\mathrm{RMN}{ }^{2} \mathrm{H}$ ), le paramètre ajustable étant la fréquence de transition $\mathrm{k}$ entre deux sites adjacents (cf. sections modèles (\$ 2.) et méthodes (\$3.1)). Les variations de $\ln (k)$ en fonction de l'inverse de la température sont reportées sur la figure 14. Sur cette figure apparaissent nettement les échelles de temps très différentes accessibles aux techniques RMN et IQNS. Il est intéressant de souligner que ces études ont été réalisées indépendamment. Ainsi l'énergie d'activation déduite des profils IQNS est de $15 \mathrm{~kJ} \mathrm{~mol}^{-1}$ et celle déduite de l'analyse des profils ${ }^{2} \mathrm{H}$ RMN de 20 $\mathrm{kJ} \mathrm{mol}^{-1}$ (cf. zooms de la figure 14). En considérant l'ensemble des données expérimentales, on trouve 19 $\mathrm{kJ}$. $\mathrm{mol}^{-1}$. Ce modèle de rotation par sauts sur 6 sites semble donc bien établi et on peut calculer les temps de relaxation $T_{1}$ théoriques correspondants. On trouve ainsi un excellent accord entre les valeurs expérimentales et théoriques en considérant une énergie d'activation de $18 \mathrm{~kJ} \cdot \mathrm{mol}^{-1}$ [9].

Dans cet exemple, l'étude combinée par IQNS et ${ }^{2} \mathrm{H}$ RMN "marche bien" et on trouve une remarquable concordance et complémentarité entre ces mesures. Nous allons maintenant aborder des cas où "ça ne marche pas si bien".

\subsection{Les $n$-paräffines}

Les n-paraffines $\mathrm{C}_{\mathfrak{n}} \mathrm{H}_{2 n+2}$ cristallisent dans des structures à feuillets et présentent généralement des phases dynamiquement désordonnées appelées "phases rotatoires" entre le cristal ordonné et le liquide. Un système prototype des $n$-paraffines est le cristal de $n$-nonadécane $\mathrm{C}_{19} \mathrm{H}_{40}$. Le cristal de $n$-nonadécane présente une seule phase ente la phase ordonnée et le liquide appelée rotatoire $\mathrm{R}_{\mathrm{I}}$. La séquence de transition est la suivante:

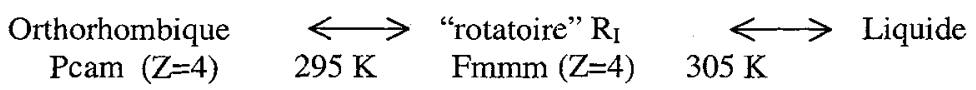

Les changements structuraux accompagnant cette transition sur schématisés sur la figure 15 .

Les spectres ${ }^{2} \mathrm{H}$ RMN sur poudre ont été réalisés [11] sur des composés sélectivement deutériés du $n$ nonadécane. Dans la phase orthorhombique de basse température, les profils correspondent à ceux attendus pour des liaisons C-D "statiques", permettant ainsi de mesurer $\chi=163 \mathrm{kHz}$ et $\eta=0$ (figure 16). Dans la phase rotatoire $\mathrm{R}_{\mathrm{I}}$, la dynamique de réorientation est rapide et le spectre obtenu présente un profil caractéristique d'une situation dans laquelle $\eta^{*} \neq 0$ (figure 16). Dans la molécule de $n$-nonadécane, toutes les liaisons C-D étant perpendiculaires au grand axe de la molécule, c'est à dire à son axe de rotation, nous pouvons conclure que la symétrie du site n'inclut pas d'axe de rotation $\mathrm{C}_{\mathrm{n}}$. Les auteurs de ces expériences [11] concluent que dans la phase rotatoire (sans autre justification), les réorientations de la molécule de $n$-nonadécane se font par des sauts sur 2 sites avec un angle $\neq \pi$. 

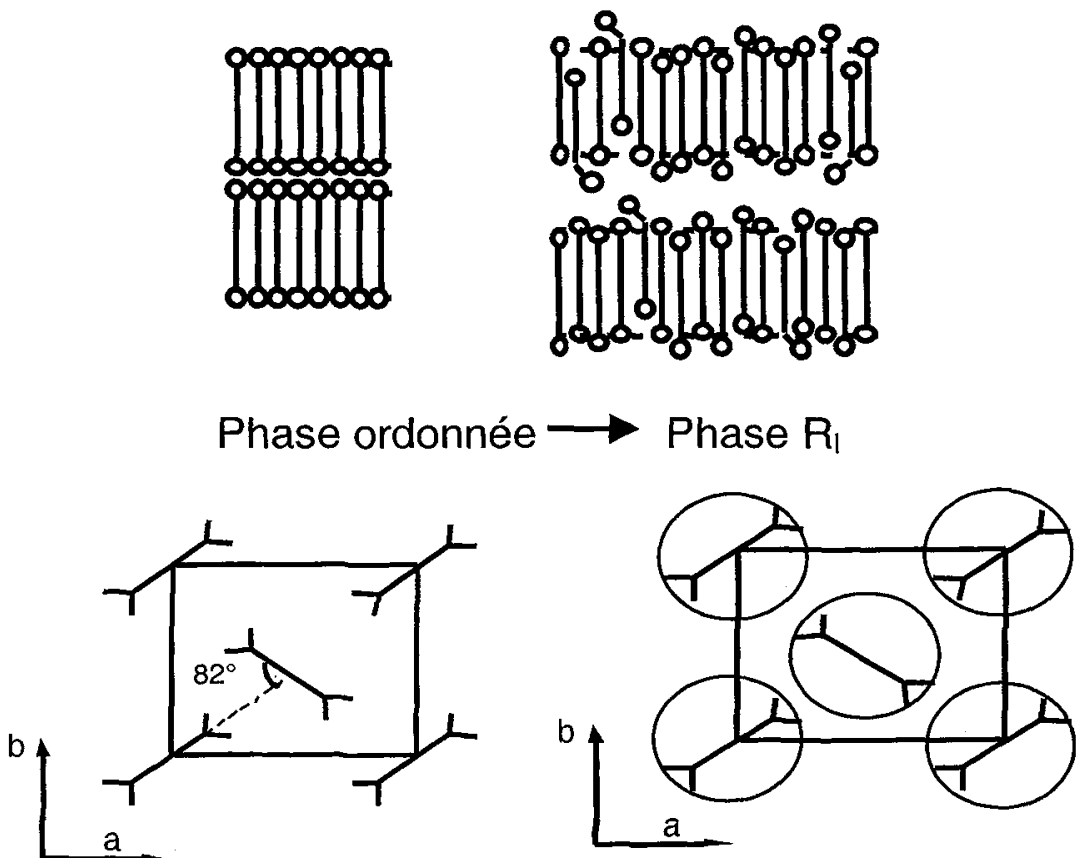

Figure 15: représentation schématique de la structure du cristal de $n$-nonadécane dans les phases ordonnée et rotatoire.

Il est facile de réaliser que pour un modèle de sauts sur 2 orientations équiprobables faisant un angle $\alpha$, nous aurons $\langle\sin (2 \phi)\rangle=\sin \alpha \cos \alpha$ et $\left\langle\cos ^{2} \phi\right\rangle=\left(1+\cos ^{2} \alpha\right) / 2$. Dans ces conditions:

$$
\begin{aligned}
& \frac{\chi^{*}}{\chi}=\frac{1}{2} \text { et } \eta^{*}=3 \cos \alpha \text { avec } \cos \alpha \leq \frac{1}{3} \\
& \frac{\chi^{*}}{\chi}=\frac{1}{4}(1+3 \cos \alpha) \text { et } \eta^{*}=3 \frac{1-\cos \alpha}{1+3 \cos \alpha} \text { avec } \frac{1}{3} \leq \cos \alpha \leq 1
\end{aligned}
$$

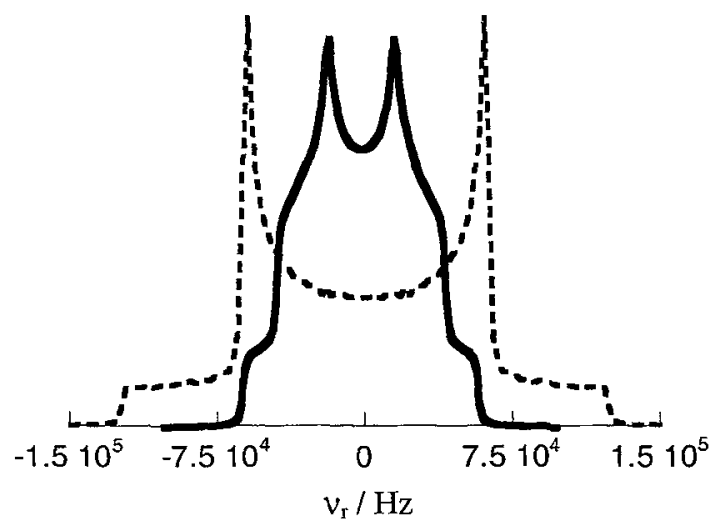

Figure 16: spectres ${ }^{2} \mathrm{H}$ RMN de poudre du $n$-nonadécane deutérié $n$ - $\mathrm{C}_{9} \mathrm{H}_{19}-\left(\mathrm{CD}_{2}\right)-\mathrm{C}_{9} \mathrm{H}_{19}$ dans la phase cristalline (pointillés) et dans la phase $R_{\text {I }}$ à $298 \mathrm{~K}$ (trait plein). 
L'examen du profil spectral montre qu'à $298 \mathrm{~K}, \chi^{*} \chi \chi=1 / 2$ et $\eta^{*}=0,40$ pour $n-\mathrm{C}_{9} \mathrm{H}_{19}-\left(\mathrm{CD}_{2}\right)-\mathrm{C}_{9} \mathrm{H}_{19}$ (figure 19), $\chi^{*} / \chi=1 / 2$ et $\eta^{*}=0,43$ pour $n-C_{19} D_{40}$. On trouve alors $\alpha=1,43$ rads $\left(82^{\circ}\right)$, valeur en accord remarquable avec l'angle que font deux molécules adjacentes dans la phase cristalline (figure 15).

Les spectres IQNS sur poudre sont complexes en raison de la coexistence de deux mécanismes de relaxation dans la phase $R_{\mathbf{r}}$. En effet, les molécules de $n$-nonadécane sont animées de mouvements de réorientation par rapport à leur grand axe et de translations restreintes le long de cet axe [12]. Afin d'analyser ces mouvements séparément, il a été nécessaire de réaliser des mosaïques de monocristaux, toutes les chaînes ayant leur axe long orienté selon l'axe perpendiculaire à la cellule de mesure, les orientations des cristallites étant aléatoires dans le plan. On peut alors orienter cette mosaïque de monocristaux par rapport au vecteur d'onde incident de manière à enregistrer des spectres IQNS avec le vecteur transfert de moment $\overrightarrow{\mathrm{Q}}$ orienté perpendiculairement aux chaînes dans une géométrie notée $\mathrm{Q}_{\perp}$ ou parallèlement à l'axe des chaînes dans la géométrie $Q_{/ l}$. Dans la géométrie $Q_{\perp}$, seules les réorientations des molécules par rapport à l'axe $\overrightarrow{\mathrm{c}}$ contribueront à l'élargissement du profil IQNS et seules les translations le long de $\overrightarrow{\mathrm{c}}$ contribueront au profil dans la géométrie $\mathrm{Q} \%$. A l'aide de ces géométries de diffusion, l'analyse des mouvements de réorientation et de translation peut être réalisée séparément. Nous allons discuter uniquement des réorientations qui sont les seuls mouvements pouvant être analysés à la fois par IQNS et ${ }^{2} \mathrm{H}$ RMN.

L'étude des spectres IQNS dans la géométrie $Q_{\perp}$ a été réalisée dans la phase $R_{I}$ à plusieurs températures (spectromètre IN6, $\lambda_{0}=5.9 \AA$, résolution $35 \mu \mathrm{eV}$ et $\lambda_{0}=5.1 \AA$, résolution $50 \mu \mathrm{eV}$ ). Vers $298 \mathrm{~K}$, l'EISF "expérimental" est assez voisin de l'EISF théorique attendu pour le modèle de rotations par sauts sur deux sites à $82^{\circ}$ (figure 17b). Notons cependant que la précision sur l'angle de saut $\alpha$ est assez mauvaise en IQNS. Les EISFs théoriques pour des angles $\alpha$ compris entre 78 et 86 degrés sont indiscernables (figure 20). Il n'en est pas du tout de même en RMN puisque le paramètre d'asymétrie $\eta^{*}$ varie de $0.6\left(\alpha=78^{\circ}\right)$ à $0.2\left(\alpha=86^{\circ}\right)$, ce qui se traduit par des profils RMN très différents (figure 7). Les EISFs "expérimentaux" sont cependant dépendant de la température (figure 17a) et les profils IQNS théoriques pour des modèles de sauts ( 2 sites ou plus) ne s'ajustent pas bien avec les profils expérimentaux. En effet, des modèles de sauts sur 2 sites prévoient une seule Lorentzienne dans l'équation (11) dont la largeur $2 \mathrm{k}$ ( $\mathrm{k}$ est la fréquence de transition) est indépendante de $\mathrm{Q}$ alors que les profils expérimentaux ont une largeur qui évolue très notablement avec Q [12].

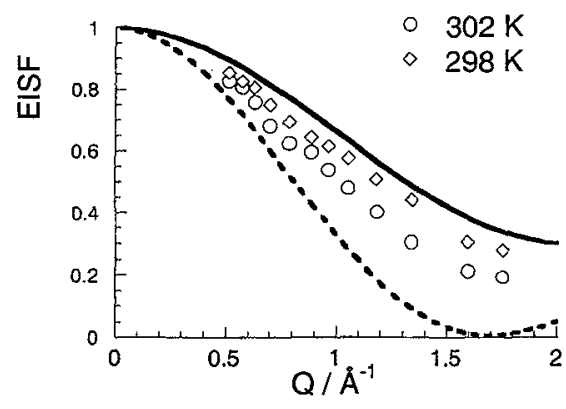

(a)

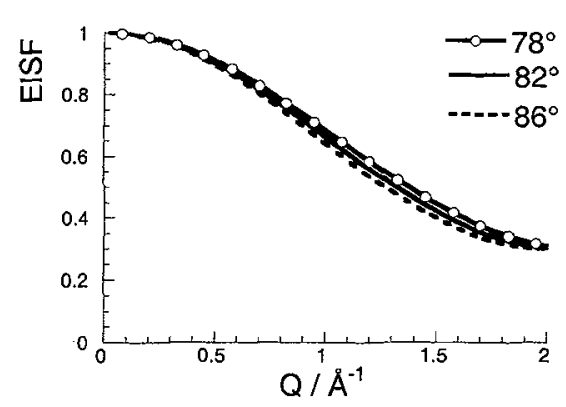

(b)

Figure 17: (a) EISF's expérimentaux (symboles) pour $n-\mathrm{C}_{19} \mathrm{H}_{40}$ dans la géométrie $\mathrm{Q}_{\perp}$ et EISFs théoriques pour des modèles de sauts sur 2 sites (trait plein, $\alpha=82^{\circ}$ ) et $N$ sites (trait continu, $N \geq 4$ ); (b) EISFs théoriques pour le modèle de sauts sur 2 orientations et plusieurs valeurs de $\alpha$. 
Ces observations suggèrent le rejet des modèles de sauts pour décrire les réorientations du $n$ nonadécane dans la phase $\mathrm{R}_{\mathrm{I}}$ au profit de modèles de rotation dans un potentiel effectif. L'expression de la fonction de distribution des orientations est, pour un modèle de diffusion dans un potentiel à deux puits séparés d'un angle $\alpha$ :

$$
\mathrm{P}(\phi)=\frac{1}{\mathrm{~F}}\left[\mathrm{e}^{-\gamma \cos \left(\phi-\frac{\alpha}{2}\right)}+\mathrm{e}^{-\gamma \cos \left(\phi+\frac{\alpha}{2}\right)}\right]
$$

L'ajustement des fonctions théoriques sur les profils expérimentaux (paramètres ajustables $\gamma, \alpha$ et le coefficient de diffusion $\mathrm{D}_{\mathrm{r}}$ ) conduit aux fonctions $\mathrm{P}(\phi)$ reportées sur la figure 18 avec $\alpha=82^{\circ}$. La comparaison des deux techniques IQNS et RMN pourrait s'arrêter cet accord qualitatif. Examinons cependant ces résultats un peu plus attentivement. A $298 \mathrm{~K}$, les spectres IQNS analysés à l'aide du modèle ci-dessus (équation (33)) conduisent à $\alpha=82^{\circ}$ (environ, compte - tenu de la relativement faible précision que la technique IQNS pour l'évaluation des angles). Dans ces conditions, nous trouvons (équations (27) (28)) $\chi^{*}=\chi / 2$ et $\eta^{*}=0.10$. A $296 \mathrm{~K}, \chi^{*}=\chi / 2$ et $\eta^{*}=0.12, \chi^{*}=\chi / 2$ et $\eta^{*}=0.07$ à $302 \mathrm{~K}$. Manifestement, le modèle de rotation diffusionnelle proposé pour analyser les données IQNS n'est pas confirmé par les mesures RMN. Ces contradictions illustrent bien les limites spécifiques de ces deux techniques:

- Les profils RMN dans la limite de modulation rapide n'offrent aucune information relative aux temps caractéristiques du mouvement. De plus, nous pourrions imaginer de nombreux modèles dynamiques autres que des sauts sur deux sites équiprobables conduisant aux valeurs de $\chi^{*}$ et $\eta^{*}$ observées.

- Les profils IQNS fournissent une information sur les temps caractéristiques du mouvement, mais à condition que ces temps soient suffisamment petits devant la résolution instrumentale. L'évolution de la largeur du profil de diffusion quasiélastique en fonction de $Q$ suggère également des modèles de rotation diffusionnelle et semblent exclure des modèles de rotations par sauts.

- Les simulations MD réalisées sur ce même système [13] ont suggéré un modèle de sauts sur 4 orientations, en apparente contradiction avec les résultats expérimentaux.

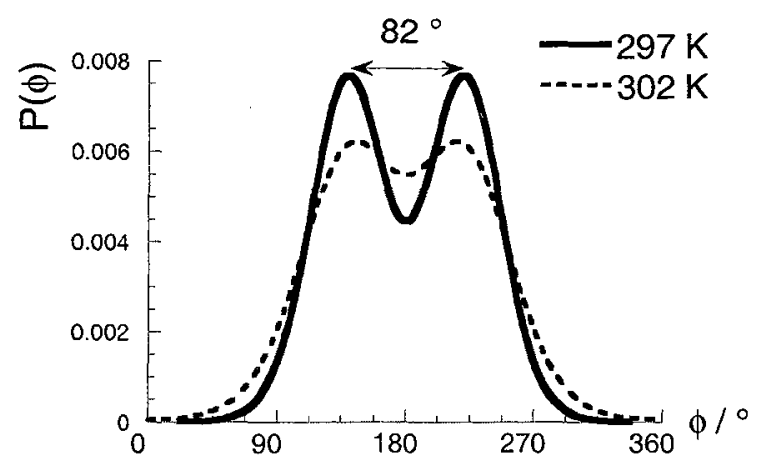

Fỉgure 18: fonctions $\mathrm{P}(\phi)$ déterminées à partir de l'ajustement des données IQNS expérimentales (IN6 à l'ILL).

Pour clarifier ce problème, une étude combinant la technique IQNS et les simulations MD a été entreprise récemment [14]. Le système MD modèle est composé de 270 molécules de $n$-nonadécane et les trajectoires atomiques calculées sur 135 ps. Les trajectoires issues de la simulation (à $300 \mathrm{~K}$ ) reflètent tous les degrés de liberté, externes (translations, rotations) et internes (torsions, vibrations). Nous appellerons ces trajectoires "tous atomes" notées TA. Des trajectoires "corps rigide" (notées CR) ont été également déterminées en ajustant une molécule dans sa conformation la plus stable "tout trans" ayant même coordonnées de centre de masse et d'orientation qu'une molécule du'système simulé. Ces 
trajectoires "corps rigide" sont sensées reproduire les mouvements de rotation et de translation des molécules à condition qu'une bonne séparation temporelle existe entre les différents degrés de liberté externes et internes.

Nous avons reporté sur la figure 19 la fonction de distribution $\mathrm{P}(\phi)$ des orientations d'une chaîne de $n$-nonadécane déterminée à partir des trajectoires CR. Cette fonction présente 4 maxima étroits et ce résultat est en contradiction flagrante avec les analyses expérimentales (figure 18). Dans une première approche, on peut supposer qu'un modèle de rotations par sauts est adéquat et il est alors possible de déterminer les constantes $\mathrm{k}$ en comptant le nombre de transitions d'un site à l'autre pendant la durée de la simulation. On obtient alors le modèle de sauts sur 4 orientations schématisé sur la figure 20 .

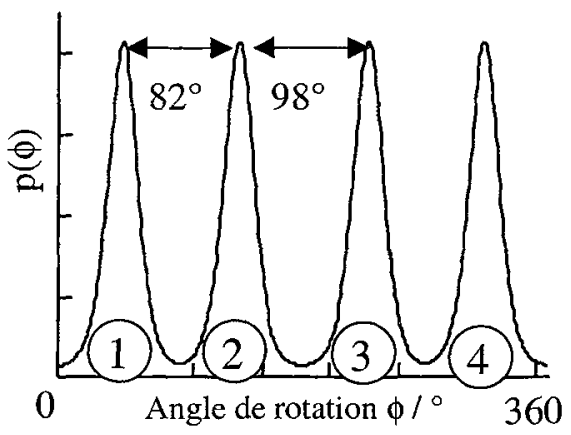

Figure 19: fonction de distribution des orientations $P(\phi)$ CR pour une chaîne de $n$-nonadécane dans la phase $R_{I}$ à $300 \mathrm{~K}$.

La première question à laquelle nous allons tenter de répondre est de savoir si ce nouveau modèle, issu de la MD, peut rendre compte des données expérimentales. Pour la RMN, il est facile de remarquer que $\langle\sin 2 \phi\rangle=\sin \alpha \cos \alpha$ et $\left\langle\cos ^{2} \phi\right\rangle=\left(1+\cos ^{2} \alpha\right) / 2$ avec $\alpha=82^{\circ}$, c'est à dire que ce modèle conduira au même profil RMN de poudre que le modèle de sauts sur 2 sites. La situation est plus complexe pour la spectroscopie IQNS puisque pour ce modèle, il nous faut considérer un élargissement du profil de diffusion quasi-élastique résultant de la somme de trois Lorentziennes de largeurs $k, k^{\prime}$ et $\left(k+k^{\prime}\right)$. Les tentatives d'ajustement des profils IQNS avec ce modèle [14] n'ont pas permis d'obtenir un accord satisfaisant entre les spectres théoriques et expérimentaux.

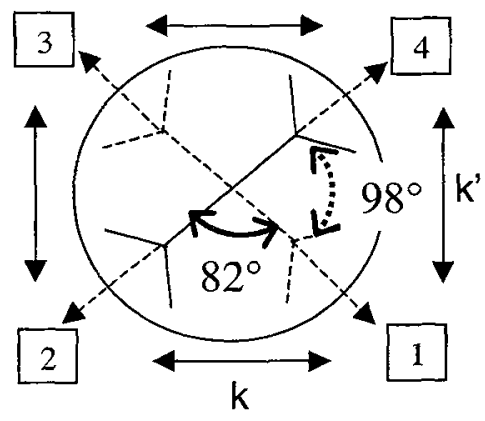

Figure 20: représentation schématique du modèle de sauts sur 4 orientations proposé à partir de l'analyse des trajectoires CR de la $\mathrm{MD}\left(\mathrm{k}=0.028 \mathrm{ps}^{-1}\right.$ et $\left.\mathrm{k}^{\prime}=0.017 \mathrm{ps}^{-1}\right)$.

Ce modèle de saut a également été utilisé pour ajuster les lois de diffusion intermédiaires calculées à partir des trajectoires $\mathrm{CR}$ de la simulation (figure 21). De toute évidence, un modèle de sauts est à exclure, même pour rendre compte des fonctions de relaxation issues de la simulation. Il convient donc de 
considérer un modèle plus élaboré de diffusion dans un potentiel à 4 puits. La fonction de distribution $\mathrm{P}(\phi)$ issue des simulations CR est parfaitement ajustée par la fonction:

$$
\mathrm{P}(\phi)=\frac{1}{\mathrm{~F}} \mathrm{e}^{-\gamma_{1} \cos 2 \phi-\gamma_{2} \cos 4 \phi}
$$

avec $\gamma_{1}=0,4$ et $\gamma_{2}=1.8$. En utilisant la méthode de discrétisation de l'équation de Fokker-Planck décrite dans la section modèles ( $\$ 2$.), on peut ajuster la loi de diffusion intermédiaire théorique aux courbes issues de la simulation avec comme paramètre le coefficient de diffusion $\mathrm{D}_{\mathrm{r}}$. L'accord parfait obtenu en suivant cette procédure est illustré par la figure 21.

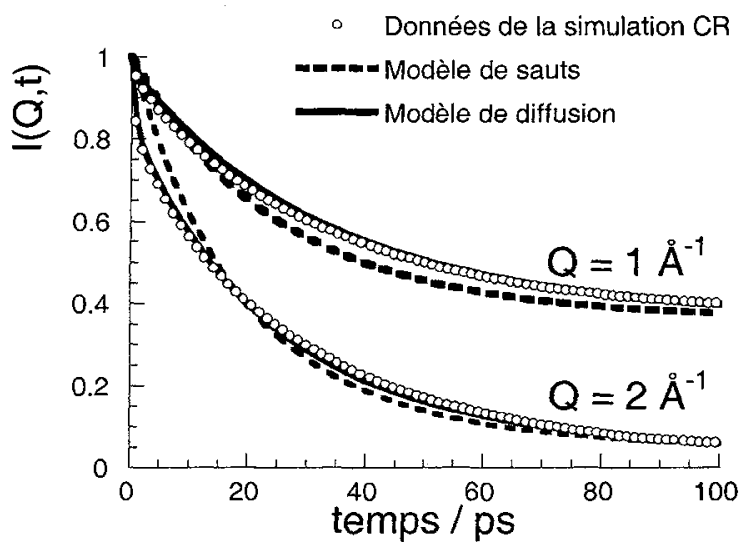

Figure 21: comparaison des modèles théoriques avec la fonction de diffusion intermédiaire calculée à partir des trajectoires atomiques $\mathrm{CR}$ de la simulation.

Que ce soit pour interpréter les données issues de la simulation ou celles issues de l'expérience IQNS, il convient donc bien de considérer un modèle relativement complexe de diffusion rotationnelle dans un potentiel à 4 puits. Le profil IQNS théorique est composé de la somme de plusieurs Lorentziennes ( 5 vers $\mathrm{Q}=2 \AA^{-1}$ ) dont la plus étroite a une $1 / 2$ largeur à $1 / 2$ intensité inférieure à $20 \mu \mathrm{eV}$. De toute évidence les conditions expérimentales précédentes (IN6 avec une résolution dont la $1 / 2$ largeur est au mieux de $35 \mu \mathrm{eV}$ [12]) ne conviennent pas et ceci explique le fait que cette composante étroite n'aie pas été détectée. De nouvelles expériences ont par conséquent été réalisées à l'ILL sur IN5 (sur des mosaïques de monocristaux) avec des résolutions instrumentales $\left(\lambda_{0}=5 \AA\right.$, résolution $40 \mu \mathrm{eV}$ et $\lambda_{0}=8 \AA$, résolution $12 \mu \mathrm{eV}$ ) plus convenables [15].

Est-ce que ce nouveau modèle convient pour rendre compte des profils RMN et IQNS? Les tentatives d'ajustement des profils théoriques calculés en fixant $\gamma_{1}=0,4$ et $\gamma_{2}=1,8\left(D_{\mathrm{r}}\right.$ étant le seul paramètre variable) sur les profils IQNS expérimentaux ont échoué, quelle que soit la température de l'échantillon. De même, en effectuant le calcul numérique des équations (26) et (27), on trouve $\chi^{*}=\chi / 2$ et $\eta^{*}=0.21$. Les ajustements des profils IQNS deviennent satisfaisants lorsque les paramètres $\gamma_{1}$ et $\gamma_{2}$ sont libres et on trouve ainsi à $296 \mathrm{~K} \gamma_{1}=0.71, \gamma_{2}=1.13\left(\mathrm{D}_{\mathrm{r}}=0.081 \mathrm{ps}^{-1}\right)$, soit $\chi^{*}=\chi / 2$ et $\eta^{*}=0.53$. A $302 \mathrm{~K}, \gamma_{1}=0.38, \gamma_{2}=0.74$ soit $\chi^{*}=\chi / 2$ et $\eta^{*}=0.37\left(\mathrm{D}_{\mathrm{r}}=0.087 \mathrm{ps}^{-1}\right)$. De toute évidence ce modèle semble convenable et permet d'interpréter les résultats issus des trois techniques. L'ajustement à l'aide de ce modèle des loịs de diffusion calculées à partir des trajectoires TA conduit à des valeurs différentes de celles obtenues avec les trajectoires CR et sont plus proches des valeurs expérimentales [15]. Ainsi $\gamma_{1}=$ 0.34 et $\gamma_{2}=1.10$ à $300 \mathrm{~K}\left(\mathrm{D}_{\mathrm{r}}=0.076 \mathrm{ps}^{-1}\right)$ ce qui donne $\chi^{*}=\chi / 2$ et $\eta^{*}=0.30$. Finalement, le profil RMN à $298 \mathrm{~K}$ peut être simulé avec ce modèle avec $\gamma_{1}=0.46$ et $\gamma_{2}=0.86$. 


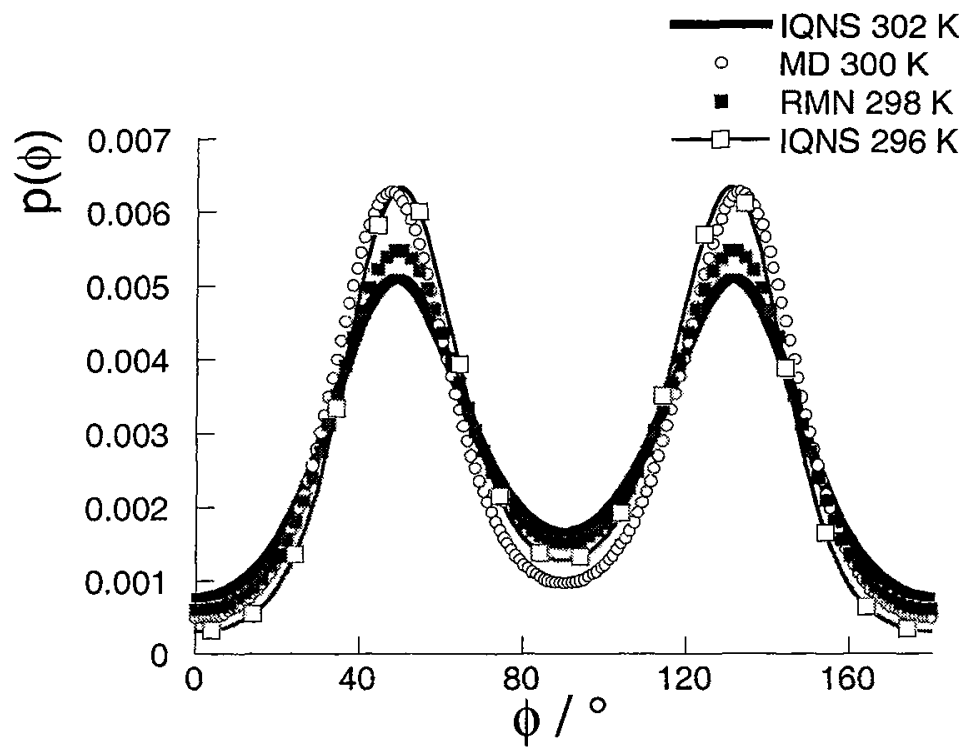

Figure 22: comparaison des fonctions de distribution des orientations $P(\phi)$ obtenues après l'ajustement des spectres IQNS, RMN et MD.

Cet exemple illustre bien l'intérêt de la démarche consistant à exploiter les simulations $\mathrm{MD}$ pour élaborer des modèles dynamiques réalistes. Sans l'apport des simulations, il aurait été beaucoup plus difficile de trouver une solution convenable permettant de réconcilier les données IQNS et RMN entre elles. Pour illustrer le bon accord obtenu, le bilan de ces résultats est donné sur la figure 23 à travers la comparaison des fonctions de distribution $\mathrm{P}(\phi)$.Notons pour conclure que nous pouvons alors déterminer les variations des barrières du potentiel de force moyenne (définies sur la figure 24) avec la température. Ainsi on trouve $\Delta \mathrm{V}_{1}=1 \mathrm{~kJ} \cdot \mathrm{mol}^{-1}$ (quelle que soit $\mathrm{T}$ ) et $2.8<\Delta \mathrm{V}_{2}<4.5 \mathrm{~kJ} \mathrm{~mol}^{-1}$ pour $296 \mathrm{~K}<\mathrm{T}<302 \mathrm{~K}$.

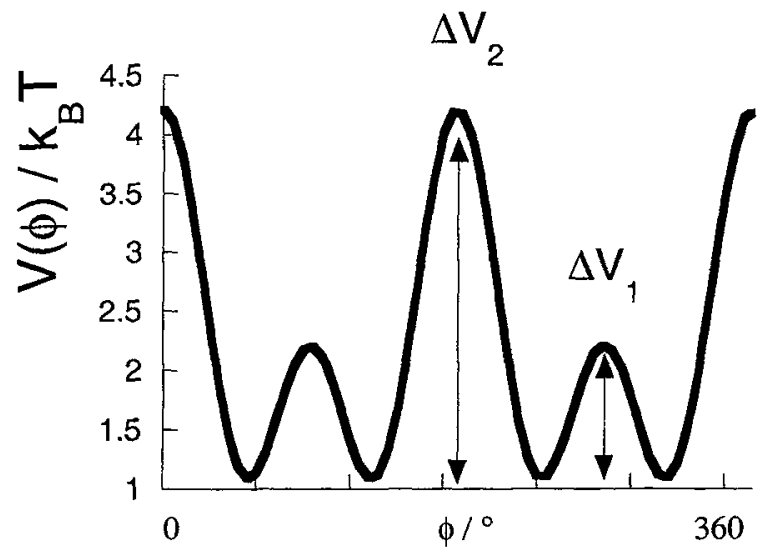

Figure 23: potentiel de force moyenne pour les réorientations du $n$-nonadécane dans la phase $\mathrm{R}_{\mathbf{I}}$.

\section{CONCLUSION}

Nous avons illustré la comparaison des techniques IQNS et ${ }^{2} \mathrm{H}$ RMN à l'aide de deux exemples:

Dans le premier (BCTC), nous avons montré combien les deux techniques sont complémentaires: 
- D'une part les échelles de temps très différentes accessibles à ces deux techniques permettent de sonder la dynamique sur une gamme de températures étendue,

- D'autre la spectroscopie IQNS permet, grâce à l'espace Q, de discriminer parmi les différents modèles plausibles pour rendre compte des profils ${ }^{2} \mathrm{H}$ RMN dans la limite de modulation rapide.

Dans le deuxième exemple, une difficulté nouvelle apparaît car les résultats IQNS et ${ }^{2} \mathrm{H}$ RMN semblent être, en première approximation, en accord (!). Cet accord n'est que très qualitatif puisque les données expérimentales issues des deux techniques ne peuvent pas être analysées à l'aide du même modèle. Dans ce cas, les simulations sur ordinateur ont été d'une aide précieuse pour construire un modèle de diffusion dans un potentiel permettant d'interpréter à la fois les spectres IQNS et ${ }^{2} \mathrm{H}$ RMN. De nombreux autres exemples de résultats contradictoires obtenus indépendamment par ces techniques spectroscopiques existent dans la littérature. Notre ambition, dans cet exposé, n'était que de souligner l'importance d'une approche multi-techniques, idéalement combinant les spectroscopies IQNS, RMN et les simulations MD. Nous pouvons citer d'autres exemples de cette complémentarité entre ces trois techniques. Par exemple nos études récentes [16] par $^{2} \mathrm{H}$ RMN et IQNS sur la dynamique du peroxyde de dioctanoyle inclus dans l'urée nous ont permis de proposer un modèle unique de rotation diffusionnelle uniaxiale décrivant la dynamique moléculaire sur une très large gamme de températures. Dans ce cas l'apport de la RMN a été capital pour préciser le nombre de puits du potentiel effectif et la technique IQNS pour en préciser la forme (les barrières).

Nous n'avons pas non plus insisté sur l'importance de l'échantillonnage pour la réalisation d'expériences de qualité. Bien que cet aspect soit hors de propos, il convient de souligner que des expériences sur des cristaux sont bien plus faciles à interpréter lorsqu'elles sont réalisées sur monocristaux que sur poudres. Nous n'avons fait que mentionner brièvement l'avantage en IQNS d'utiliser des monocristaux pour identifier et analyser les translations moléculaires dans des phases solides. Il est également important de souligner la précision considérable que nous pouvons obtenir en ${ }^{2} \mathrm{H}$ RMN sur les distributions d'orientations (statiques ou dynamiques) en utilisant des monocristaux.

\section{Remerciements}

Je remercie avec plaisir ceux qui m'ont tant apporté dans les domaines de la spectroscopie IQNS; A.-J. Dianoux (I.L.L. Grenoble), M. Bée (Université J. Fourier, Grenoble), de la spectroscopie RMN; E.J. Dufourc (C.R.P.P. Talence), B. Toudic (GMCM Université de Rennes), K.D.M. Harris (Université de Birmingham); des simulations numériques; J.C. Smith, M. Souaille (C.E. Saclay), J.-P. Ryckaert (Université Libre de Bruxelles).

\section{Références}

1. W. Nadler, K. Schulten, J. Chem. Phys., 844015 (1986).

2. G. Coddens (dans ce journal).

3. M. Bée (dans ce journal).

4. P. Damay (dans ce journal)

5. A. Abragam in The principles of Nuclear Magnetism (Oxford University Press, London, 1961).

6. R.J. Wittebort, E.T. Olejniczak, R.G. Griffin, J. Chem. Phys. 86, 5411 (1987).

7. D.A. Torchia, A. Szabo, J. Magn. Reson. 49107 (1982).

8. G.J. Kearley, B. Nicolaï (dans ce journal).

9. A.E. Aliev, K.D.M. Harris, F. Guillaume, J. Phys. Chem, 99, 1156 (1995).

10. G. Lucazeau, H. Chor, C. Sourisseau, A.J. Dianoux, J. Chem.Phys., 76, 307 (1983). 
11. M.G. Taylor, E.C. Kelusky, I.C.P. Smith, H.L. Casal, D.G. Cameron, J. Chem. Phys. 78, 5108 (1983).

12. F. Guillaume, J. Doucet, C. Sourisseau, A.-J. Dianoux, J. Chem. Phys. 91, 2555 (1989).

13. J.-P. Ryckaert, M. L. Klein, I. R. McDonald, Mol. Phys. 83, 439 (1994).

14. L. Gonzalez Mac Dowell, F. Guillaume, J.-P. Ryckaert, P. Girard, V. Rodriguez, A.-J. Dianoux, Physica B. 234 - 236, 106 (1997).

15. F. Guillaume, J.-P. Ryckaert, V. Rodriguez, L. Gonzalez Mac Dowell, P. Girard, ‘A.-J. Dianoux (en préparation)

16. P. Girard, A.E. Aliev, F. Guillaume, K.D.M. Harris, M.D. Hollingsworth, A.-J. Dianoux, P. Jonsen, J. Chem. Phys., 109, 4078 (1998). 